Article

\title{
Design, Synthesis and Biological Evaluation of 6,7-Disubstituted-4-phenoxyquinoline Derivatives Bearing Pyridazinone Moiety as c-Met Inhibitors
}

\author{
Xiaobo Liu ${ }^{1}$, Jianlan Kou ${ }^{2}$, Zhen Xiao ${ }^{1}$, Fajuan Tian ${ }^{1}$, Jiayi Hu ${ }^{1}$, Pengwu Zheng ${ }^{1, *}$ \\ and Wufu Zhu 1,* (D) \\ 1 Jiangxi Provincial Key Laboratory of Drug Design and Evaluation, School of Pharmacy, \\ Jiangxi Science \& Technology Normal University, Nanchang 330013, China; 18790658261@163.com (X.L.); \\ xz950420@163.com (Z.X.); 15679170508@163.com (F.T.); 15797865216@163.com (J.H.) \\ 2 Teaching and Research Department of Mathematics and Chemistry, Nanchang Health School, \\ Nanchang 330006, China; hjy15717910426@163.com \\ * Correspondence: zhengpw@jxstnu.edu.cn (P.Z.); zhuwf@jxstnu.edu.cn (W.Z.); \\ Tel./Fax: +86-791-8380-2393 (W.Z.)
}

Received: 1 June 2018; Accepted: 19 June 2018; Published: 26 June 2018

\begin{abstract}
Deregulation of the receptor tyrosine kinase mesenchymal epithelial transition factor (MET) has been implicated in several human cancers and is an attractive target for small molecule drug discovery. Herein, a series of 6,7-disubstituted-4-phenoxyquinoline derivatives bearing pyridazinone derivatives were designed, synthesized and evaluated for their enzymatic inhibitory activity against c-Met kinase and cellular potency against A549, HepG2, and MCF-7 cell lines. Eight of them are equal to more active than positive control Foretinib against one or more cell lines and enzyme. The most promising compound 53 showed superior activity to Foretinib, which possessed excellent c-Met kinase inhibition on a singledigital nanomolar level $\left(\mathrm{IC}_{50}=0.6 \mathrm{nM}\right)$, and cancer cells of A549 $\left(\mathrm{IC}_{50}=0.003 \mu \mathrm{M}\right)$, HepG2 $\left(\mathrm{IC}_{50}=0.49 \mu \mathrm{M}\right)$ and MCF-7 cells $\left(\mathrm{IC}_{50}=0.006 \mu \mathrm{M}\right)$. The result of AO single staining indicated that compound 53 could induce remarkable apoptosis of HepG2 cell.
\end{abstract}

Keywords: quinoline derivatives; pyridazinone; c-Met; antiproliferative activity

\section{Introduction}

The c-mesenchymal-epithelia transition factor (c-Met), known as hepatocyte growth factor receptor (HGFR), belongs to the receptor tyrosine kinases (RTKs) subfamily [1,2]. The MET receptor tyrosine kinase is activated by binding to its ligand HGF, resulting in the dimerization of the receptor. Then, Tyr1234/1235 is autophosphorylated, and activates the kinase domain and downstream multiple signaling pathways (including RAS/MAPK and PI3K/AKT), which promotes cell proliferation, survival, movement, invasion, angiogenesis and morphogenesis [3,4]. Aberrant c-Met signaling activation due to gene amplification, rearrangement, point mutations, and autocrine or paracrine HGF stimulation has been implicated in many types of human malignancies [5-9]. In addition, $\mathrm{HGF}$ /c-Met over-activation is involved in mediating intrinsic or acquired resistance to target therapies. For example, amplification of the Met has been detected in 20\% of acquired resistance to epidermal growth factor receptor (EGFR) inhibitors [10,11]. Therefore, the c-Met axis has emerged as an attractive target for targeted cancer therapy.

In recent years, significant progress has been made in the development of small molecule c-Met kinase inhibitors. Cabozantinib (1 XL-184) is the first multitarget small molecule c-Met kinase inhibitor approved by the FDA for the treatment of medullary thyroid carcinoma on 29 November 2012 [12]. Foretinib (2 GSK1363089), the first small molecule oral inhibitor to enter clinical trials, is in clinical stage 
II currently [13]. Many other kinds of c-Met inhibitor are also reported, such as TAS-115, Altiratinib, Merestinib, BMS-777607 (3-6, Figure 1) [14-18].

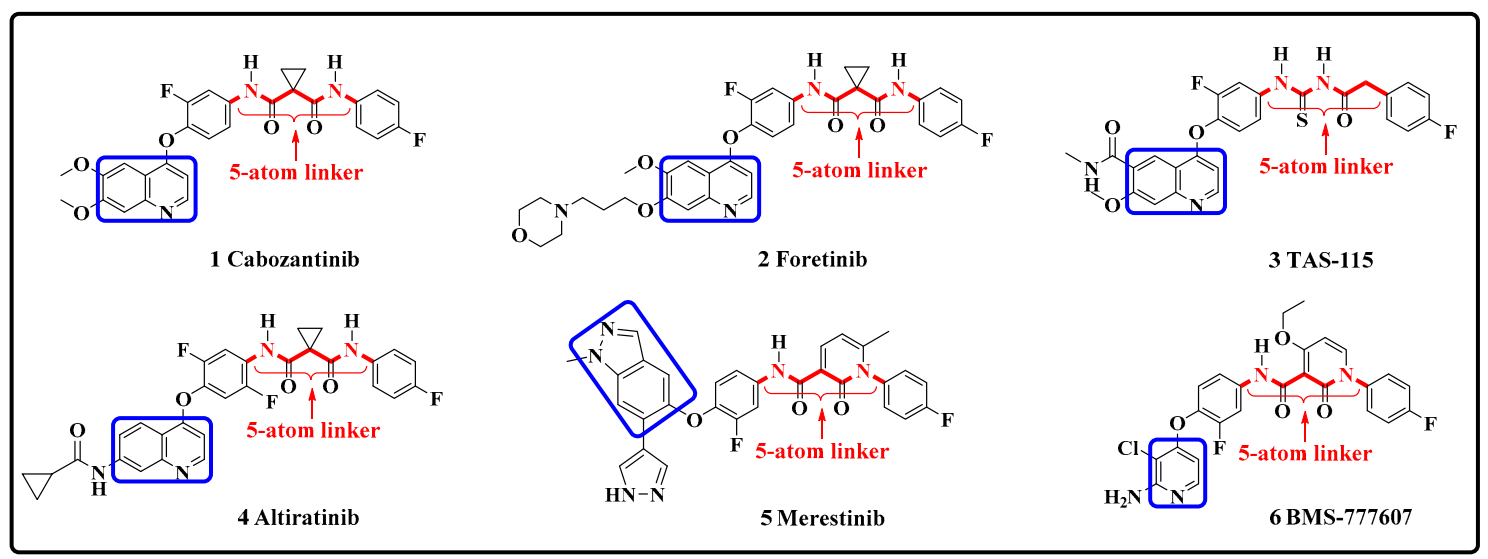

Figure 1. The representative small-molecule c-Met kinase inhibitors.

Because of its excellent efficacy in vivo, favorable pharmacokinetics and preclinical safety profiles, Foretinib was chosen as an extension of our work on the development of novel potent c-Met inhibitors. And many researches have shown that almost all Foretinib derivatives containing 5 -atom linker between the aminophenoxy at C-4 position of quinoline and aryl group, which we called "5-atom regulation" exhibit excellent activity. The '5-atom regulation' have two obvious structural characteristics. One is that there is six chemical bonds distance between aminophenoxy at C-4 position of quinoline moiety and aryl group moiety, and the other one is that the "six chemical bonds linker" contains hydrogen, oxygen, and nitrogen atoms which could form hydrogen-bond donor or acceptor (Figure 1) [19].

In our previous research, most of these compounds showed potent activity, especially the most promising compound 7 (Figure 2) with the enzyme activity $\mathrm{IC}_{50}$ values in the nanomole level [20]. The SARs and docking study exhibited that 6-oxo-pyridazinone moiety may be benefit to the in vitro activity. From the 3D model of Foretinib, we can know that Foretinib contain an intermolecular hydrogen (distance $=1.64 \mathrm{~A}^{\circ}$ ) in the 5-atom linker. And inspired by compound 7, 4-oxo-pyridazinone was introduced into 5-atom linker moiety to limit the conformation deeply.

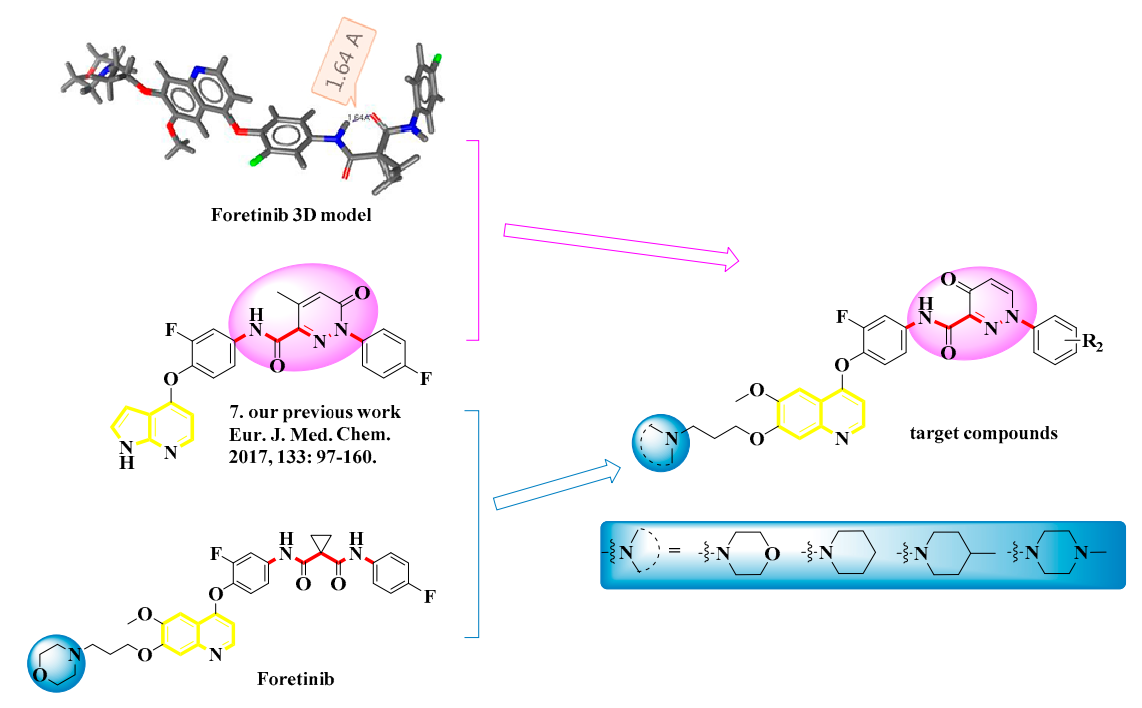

Figure 2. Structures and design strategy for target compounds. 
The biological activity of compound 7 incorporating pyrrolo[2,3-b]pyridine was not optimal. Therefore, reference was made to the target compound Foretinib, which retains the 3-carbon tether at the 7 position of quinolone. In order to further increase the solubility of the compound to increase activity, the morpholinyl group was replaced by other water-soluble substituents, including piperidinyl, 4-methylpiperidinyl and 4-methylpiperazine, to observe the effects of the different cyclic tertiary amino groups on activity of the novel compounds. Furthermore, various substituents $\left(\mathrm{R}_{2}\right)$ were introduced at the phenyl ring to investigate their effects on activity. Accordingly, we designed a novel series of 6,7-disubstituted-4-phenoxy quinoline derivatives bearing pyridazinone moiety.

The target compounds were evaluated for their enzymatic inhibitory activity against c-Met kinase and cellular potency in human liver cancer (HepG2), human breast cancer (MCF-7) and human lung adenocarcinoma (A549) cell lines [21]. Moreover, AO single staining and docking studies were presented in this paper as well.

\section{Results and Discussion}

The synthesis of the key intermediates of 6,7-disubstituted-4-phenoxyquinolines 15a-d was illustrated in Scheme 1. The commercially available starting material 1-(4-hydroxy-3-methoxyphenyl) ethanone was alkylated with 1-bromo-3-chloropropane in acetone under basic condition to provide compound 8 , which was converted to nitro compound 9 using fuming nitric acid as nitration reagent in dichloromethane at $-20^{\circ} \mathrm{C}$ for $6 \mathrm{~h}$. Next, condensation of 9 with dimethyl formamide dimethyl acetal (DMF-DMA) in refluxing toluene afforded yellow solid 10, which was reduced and cyclized using glacial acetic acid and iron powder to provide hydroxy-quinoline $\mathbf{1 1}$ with a high yield and purity in a single step. Substitution of $\mathbf{1 1}$ with excessive secondary amines (piperidine, 4-methyl piperidine, morpholine, and pyrrolidine) in acetonitrile at reflux provided intermediates $\mathbf{1 2 a}-\mathbf{d}$, which were treated with phosphorus oxychloride to afford chloro-quinoline 13a-d, respectively. Further, the obtained compounds 13a-d were etherified with 2-fluoro-4-nitrophenol in chlorobenzene to obtain purified compounds 14a-d, which were reduced using iron powder and catalytic amounts of ammonium chloride in ethanol to obtain amides 15a-d [22].

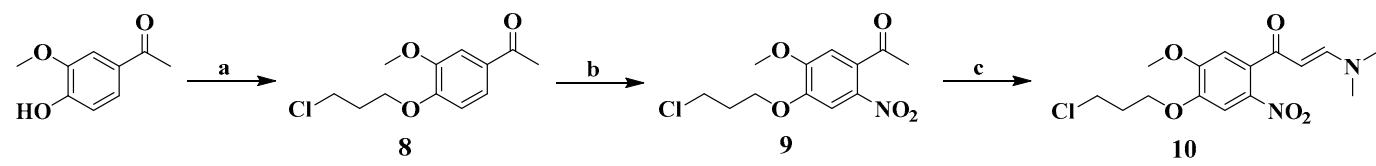

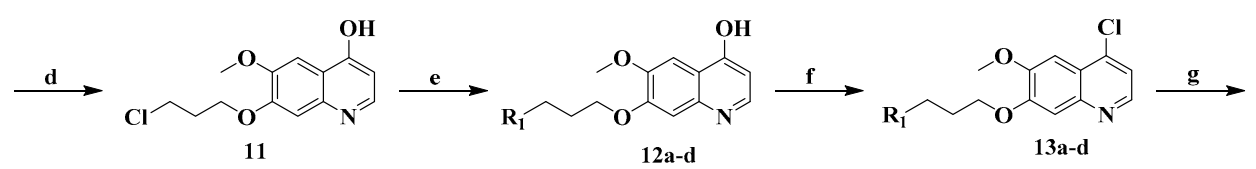

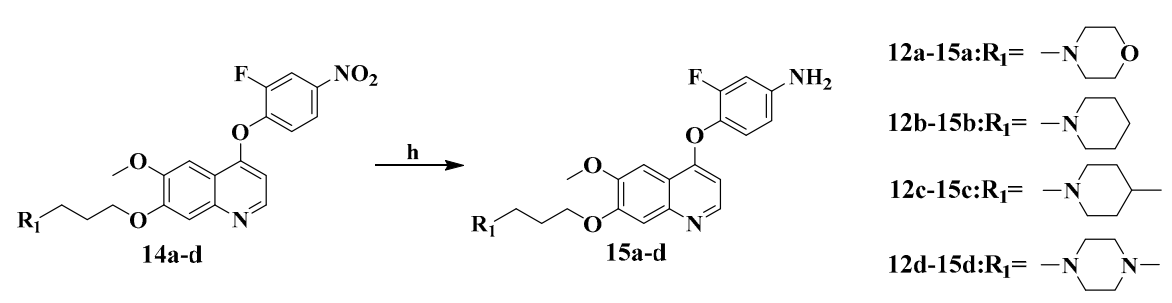

Scheme 1. Reagents and conditions: (a) $\mathrm{Br}\left(\mathrm{CH}_{2}\right)_{3} \mathrm{Cl}$, acetone, $0{ }^{\circ} \mathrm{C}, 30 \mathrm{~min}, \mathrm{rt}, 12 \mathrm{~h}$; (b) $98 \% \mathrm{HNO}_{3}$, $\mathrm{CH}_{2} \mathrm{Cl}_{2}, 0^{\circ} \mathrm{C}, 4 \mathrm{~h}$; (c) DMF-DMA, toluene, $110{ }^{\circ} \mathrm{C}, 10 \mathrm{~h}$; (d) Fe powder, $\mathrm{AcOH}$, rt, $30 \mathrm{~min}, 80^{\circ} \mathrm{C}, 2 \mathrm{~h}$; (e) secondary amines, $\mathrm{CH}_{3} \mathrm{CN}, 85^{\circ} \mathrm{C}, 10 \mathrm{~h}$; (f) $\mathrm{POCl}_{3}, 85^{\circ} \mathrm{C}, 6 \mathrm{~h}$; (g) 2-fluoro-4-nitrophenol, $\mathrm{PhCl}$, $140{ }^{\circ} \mathrm{C}, 30 \mathrm{~h}$; (h) Fe powder, $\mathrm{NH}_{4} \mathrm{Cl}$ (cat.), EtOH $/ \mathrm{H}_{2} \mathrm{O}$, reflux, $5 \mathrm{~h}$.

The target compounds 22-53 were prepared as illustrated in Scheme 2 Substituted anilines 16a-h were diazotized and then reacted with ethyl aceto-acetate to get compounds $\mathbf{1 7} \mathbf{a}-\mathbf{h}$, which condensed 
with DMF-DMA to yield compounds 18a-h. Following by cyclization reaction, hydrolysis reaction and chlorination reaction, $\mathbf{2 1} \mathbf{a}-\mathbf{h}$ were obtained. Finally, reaction of amides $\mathbf{1 5 a} \mathbf{-} \mathbf{d}$ with acyl chlorides $\mathbf{2 1 a}-\mathbf{h}$ promoted by DIPEA in dichloromethane at room temperature yielded the target compounds 22-53.

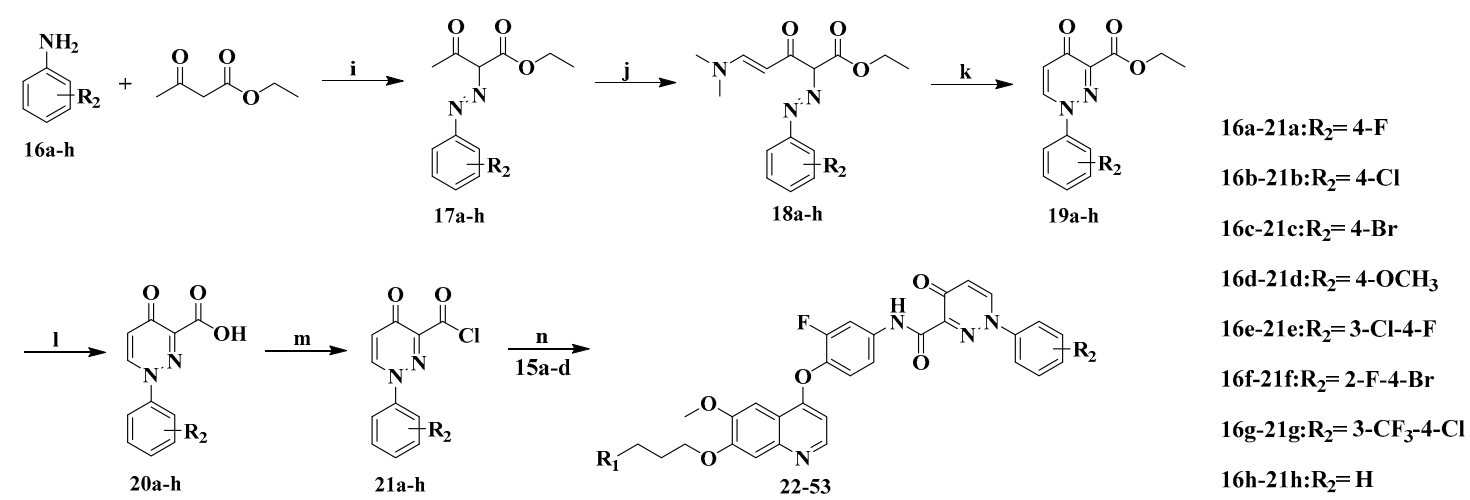

Scheme 2. Reagents and conditions: (i) sodium nitrite, $\mathrm{EtOH}$, ethyl acetoactate, $0{ }^{\circ} \mathrm{C}, 30 \mathrm{~min}$; (j) DMF-DMA, $100{ }^{\circ} \mathrm{C}$, reflux, 6-10 h; (k) EtOH, $10 \% \mathrm{NaOH}, 80{ }^{\circ} \mathrm{C}, 1 \mathrm{~h}$; (l) sodium carbonate, $\mathrm{EtOH} / \mathrm{H}_{2} \mathrm{O}$ (5:1), $80{ }^{\circ} \mathrm{C}$, 3-5 h; (m) oxalyl chloride, DMF, $\mathrm{CH}_{2} \mathrm{Cl}_{2}, \mathrm{rt}, 5 \mathrm{~min}$; (n) DIPEA, $\mathrm{CH}_{2} \mathrm{Cl}_{2}, \mathrm{rt}, 0.5 \mathrm{~h}$.

\subsection{Biological Evaluation}

Taking Foretinib as reference compound, the target compounds (22-53) were evaluated for the activity against c-Met kinase and the cytotoxicity against three cancer cell lines A549, HepG2, MCF-7 by 3-(4,5-dimethylthiazolyl-2)-2, 5-diphenyltetrazolium bromide (MTT) cell proliferation assay. The results expressed as $\mathrm{IC}_{50}$ values were summarized in Table 1 , and the values were the average of at least two independent experiments.

Table 1. c-Met Enzymatic and Cellular Activities of the target compounds 22-53.

\begin{tabular}{|c|c|c|c|c|c|c|}
\hline \multirow{2}{*}{ Compounds } & \multirow{2}{*}{$\mathbf{R}_{\mathbf{1}}$} & \multirow{2}{*}{$\mathbf{R}_{\mathbf{2}}$} & \multirow{2}{*}{$\begin{array}{c}\mathrm{IC}_{50}{ }^{\mathrm{a}}(\mathrm{nM}) \\
\mathrm{c}-\mathrm{Met}\end{array}$} & \multicolumn{3}{|c|}{$\mathrm{IC}_{50}(\mu \mathrm{M})$} \\
\hline & & & & A549 & HepG2 & MCF-7 \\
\hline 22 & & $4-F$ & 47.0 & $0.25 \pm 0.01$ & $2.23 \pm 0.35$ & $0.21 \pm 0.02$ \\
\hline 23 & & $4-\mathrm{Cl}$ & $>500$ & $N^{b}$ & NA & NA \\
\hline 24 & & $4-\mathrm{Br}$ & $>500$ & NA & NA & NA \\
\hline 25 & & $4-\mathrm{OCH}_{3}$ & 233.0 & $4.24 \pm 0.62$ & $6.75 \pm 0.82$ & $27.24 \pm 1.44$ \\
\hline 26 & & $3-\mathrm{Cl}-4 \mathrm{~F}$ & 352.0 & $3.70 \pm 0.57$ & NA & $5.96 \pm 0.77$ \\
\hline 27 & & $2-\mathrm{F}-4 \mathrm{Br}$ & 478.0 & NA & NA & NA \\
\hline 28 & & $3-\mathrm{CF}_{3}-4 \mathrm{Cl}$ & 382.0 & $5.25 \pm 0.72$ & NA & $7.49 \pm 0.87$ \\
\hline 29 & & $\mathrm{H}$ & 16.0 & $0.20 \pm 0.02$ & $2.27 \pm 0.36$ & $0.03 \pm 0.01$ \\
\hline 30 & & $4-\mathrm{F}$ & 17.0 & $0.31 \pm 0.01$ & $0.99 \pm 0.01$ & $0.69 \pm 0.02$ \\
\hline 31 & & $4-\mathrm{Cl}$ & 78.0 & $0.57 \pm 0.02$ & $0.69 \pm 0.03$ & $1.16 \pm 0.06$ \\
\hline 32 & & $4-\mathrm{Br}$ & 246.0 & $2.89 \pm 0.46$ & $1.75 \pm 0.24$ & $2.45 \pm 0.39$ \\
\hline 33 & & $4-\mathrm{OCH}_{3}$ & 56.0 & $2.78 \pm 0.44$ & $0.63 \pm 0.08$ & $2.35 \pm 0.37$ \\
\hline 34 & & $3-\mathrm{Cl}-4 \mathrm{~F}$ & 2.0 & $0.36 \pm 0.04$ & $1.28 \pm 0.11$ & $0.15 \pm 0.04$ \\
\hline 35 & & $2-\mathrm{F}-4 \mathrm{Br}$ & 8.0 & $1.36 \pm 0.19$ & $3.10 \pm 0.49$ & $1.19 \pm 0.08$ \\
\hline 36 & & $3-\mathrm{CF}_{3}-4 \mathrm{Cl}$ & 11.0 & $0.79 \pm 0.06$ & $1.80 \pm 0.26$ & $0.73 \pm 0.08$ \\
\hline 37 & & $\mathrm{H}$ & 4.0 & $0.06 \pm 0.01$ & $0.18 \pm 0.01$ & $0.03 \pm 0.01$ \\
\hline
\end{tabular}


Table 1. Cont.

\begin{tabular}{|c|c|c|c|c|c|c|}
\hline \multirow{2}{*}{ Compounds } & \multirow{2}{*}{$\mathbf{R}_{\mathbf{1}}$} & \multirow{2}{*}{$\mathbf{R}_{2}$} & \multirow{2}{*}{$\begin{array}{c}\mathrm{IC}_{50}{ }^{\mathrm{a}}(\mathrm{nM}) \\
\mathrm{c}-\mathrm{Met}\end{array}$} & \multicolumn{3}{|c|}{$\mathrm{IC}_{50}(\mu \mathrm{M})$} \\
\hline & & & & A549 & HepG2 & MCF-7 \\
\hline 38 & & $4-\mathrm{F}$ & $>500$ & $28.89 \pm 1.46$ & $47.23 \pm 1.67$ & $22.47 \pm 1.35$ \\
\hline 39 & & $4-\mathrm{Cl}$ & 169.0 & $12.43 \pm 1.09$ & $7.81 \pm 0.89$ & $14.14 \pm 1.15$ \\
\hline 40 & & $4-\mathrm{Br}$ & 345.0 & $13.47 \pm 1.13$ & $8.84 \pm 0.95$ & $9.74 \pm 0.99$ \\
\hline 41 & & $4-\mathrm{OCH}_{3}$ & 0.9 & $0.02 \pm 0.01$ & $0.008 \pm 0.001$ & $0.02 \pm 0.01$ \\
\hline 42 & & $3-\mathrm{Cl}-4 \mathrm{~F}$ & 1.2 & $0.005 \pm 0.001$ & $1.03 \pm 0.01$ & $0.007 \pm 0.001$ \\
\hline 43 & & $2-\mathrm{F}-4 \mathrm{Br}$ & 2.0 & $0.03 \pm 0.01$ & $0.003 \pm 0.001$ & $0.005 \pm 0.001$ \\
\hline 44 & & $3-\mathrm{CF}_{3}-4 \mathrm{Cl}$ & 19.0 & $0.15 \pm 0.03$ & $0.65 \pm 0.05$ & $5.96 \pm 0.07$ \\
\hline 45 & & $\mathrm{H}$ & 187.0 & $2.95 \pm 0.47$ & $4.66 \pm 0.67$ & $2.01 \pm 0.30$ \\
\hline 46 & & $4-\mathrm{F}$ & 39.0 & $1.64 \pm 0.22$ & $7.51 \pm 0.87$ & $1.66 \pm 0.22$ \\
\hline 47 & & $4-\mathrm{Cl}$ & 145.0 & $19.99 \pm 1.30$ & $14.25 \pm 1.15$ & $21.08 \pm 1.32$ \\
\hline 48 & & $4-\mathrm{Br}$ & $>500$ & NA & NA & NA \\
\hline 49 & & $4-\mathrm{OCH}_{3}$ & 78.0 & $9.12 \pm 0.96$ & $3.68 \pm 0.57$ & $8.12 \pm 0.91$ \\
\hline 50 & & $3-\mathrm{Cl}-4 \mathrm{~F}$ & 2.0 & $0.02 \pm 0.01$ & $2.46 \pm 0.39$ & $0.02 \pm 0.01$ \\
\hline 51 & & $2-\mathrm{F}-4 \mathrm{Br}$ & 123.0 & $5.63 \pm 0.75$ & $6.39 \pm 0.81$ & $5.43 \pm 0.74$ \\
\hline 52 & & $3-\mathrm{CF}_{3}-4 \mathrm{Cl}$ & 0.8 & $0.04 \pm 0.01$ & $0.33 \pm 0.03$ & $0.15 \pm 0.02$ \\
\hline 53 & & $\mathrm{H}$ & 0.6 & $0.003 \pm 0.001$ & $0.49 \pm 0.03$ & $0.006 \pm 0.001$ \\
\hline Foretinib $^{c}$ & - & - & 1.8 & $0.26 \pm 0.03$ & $0.84 \pm 0.07$ & $2.76 \pm 0.53$ \\
\hline
\end{tabular}

As illustrated in Table 1, most target compounds showed moderate to significant cytotoxic activities against one or more tested cancer cells with potencies in the single digit micromole range, which suggested that the introduction " 5 -atom linker" to 4-oxo-pyridazinone framework maintained the potent cytotoxic activity. Six compounds $(37,41,42,43,52$ and 53) exhibited promising cytotoxicity with $\mathrm{IC}_{50}$ values ranging from 0.002 to $1.03 \mu \mathrm{M}$, which was comparable to that of Foretinib. It is worth noting that the most significant candidate compound $\mathbf{5 3}$ showed significant activity with $\mathrm{IC}_{50}$ values of $0.003 \mu \mathrm{M}, 0.49 \mu \mathrm{M}, 0.006 \mu \mathrm{M}$ for A549, HepG2 and MCF-7 cells, respectively, which were higher than those of Foretinib against A549 cell $(0.26 \mu \mathrm{M})$, HepG2 cell $(0.84 \mu \mathrm{M})$, MCF-7 cell $(2.76 \mu \mathrm{M})$.

Target compounds were further determined for c-Met kinase activity through homogenous time-resolved fluorescence (HTRF) assays. As shown in Table 1, most target compounds exhibited moderate to excellent c-Met enzymatic potency with $\mathrm{IC}_{50}$ values ranging, suggesting that the inhibition of c-Met may be a main mechanism for the antitumor activity of the prepared compounds. Four compounds $41(0.9 \mathrm{nM}), \mathbf{4 2}(1.2 \mathrm{nM}), \mathbf{5 2}(0.8 \mathrm{nM})$ and $\mathbf{5 3}(0.6 \mathrm{nM})$ exhibited promising enzymatic activity, which were more active than reference compound Foretinib $(1.8 \mathrm{nM})$.

The structure-activity relationship (SAR) was commenced by the morpholinyl group was replaced by other water-soluble substituents, including piperidinyl, 4-methylpiperidinyl and 4-methylpiperazine. The pharmacological activity data indicated that the $\mathrm{R}_{1}$ group affected the activity dramatically. The compounds (22-29) substituted with morpholine have slight reduced the cytotoxicity activity against A549, HepG-2 and MCF-7 cell lines than the compounds (30-53) substituted with other three water-soluble groups (piperidine, 4-methylpiperidine and 4-methylpiperazine). Furthermore, the cell lines data revealed a clear preference for activity when the $\mathrm{R}_{1}$ group was 4-methyl piperazinyl group, which indicated that a more water-soluble cyclic tertiary amino group at the C-7 position of quinoline contributed to the potency of the target compounds. Such as, the $\mathrm{IC}_{50}$ values of compound 52 were $(0.8 \mathrm{nM}, 0.04 \mu \mathrm{M})$ against c-Met kinases and A549 cell lines, which were clearly lower than that of compound $28(382.0 \mathrm{nM}, 5.25 \mu \mathrm{M})$, compound $36(11.0 \mathrm{nM}, 0.79 \mu \mathrm{M})$ and compound $44(19.0 \mathrm{nM}$, $0.15 \mu \mathrm{M})$.

Further investigations were performed to study the effect of different substituents on the phenyl ring on the cytotoxic activity. In general, it seems to be that target compounds with no less than one electron-withdrawing groups (EWGs) showed better in vitro activity, and the aryl group 
substituted with double-EWGs is more preferred. For instance, the enzymatic potency of double-EWGs compounds $34(2.0 \mathrm{nM}), 35(8.0 \mathrm{nM})$ and $36(11.0 \mathrm{nM})$, were clearly lower than that of mono-EWGs compounds $30(17.0 \mathrm{nM}), 31(78.0 \mathrm{nM})$ and $32(246.0 \mathrm{nM})$. These data also showed that when there is only one EWGs (4-F, 4-Cl, 4-Br), the compounds substituted with the substituent of increasing electrophilic ability were beneficial to the antiproliferative activity. In addition, compounds without substituent group on aryl group exhibit the best activity, such as compounds 29, 37 and 53. However, compound 45 is an exception, compounds substituted with the substituent of increasing electrophilic ability were beneficial to the antiproliferative activity.

\subsection{Acridine Orange (AO) Staining Is Used to Analyze Cell Morphological Changes of the HepG2 Cells}

Potential compound 53 was selected for validation and evaluation of inhibition of cell proliferation and induction of apoptosis in HepG2 cells. AO staining fluorescence analysis results showed that compared to the control group (Figure 3a), compound 53 (Figure 3b) significantly inhibited HepG2 cells proliferation and induced apoptosis at the concentration of $0.01 \mu \mathrm{M}$. Moreover, from the results of the inverted fluorescence microscopy, it can be observed that the cells in the control group (Figure 3a) were full and the edge was clear, which was added without nothing. And HepG2 cells were treated with compound 53, it can be seen in (Figure 3b) that the HepG2 cells showed obviously shrink into chromatin, with sharp edges and consistent with the phenomenon of apoptosis.

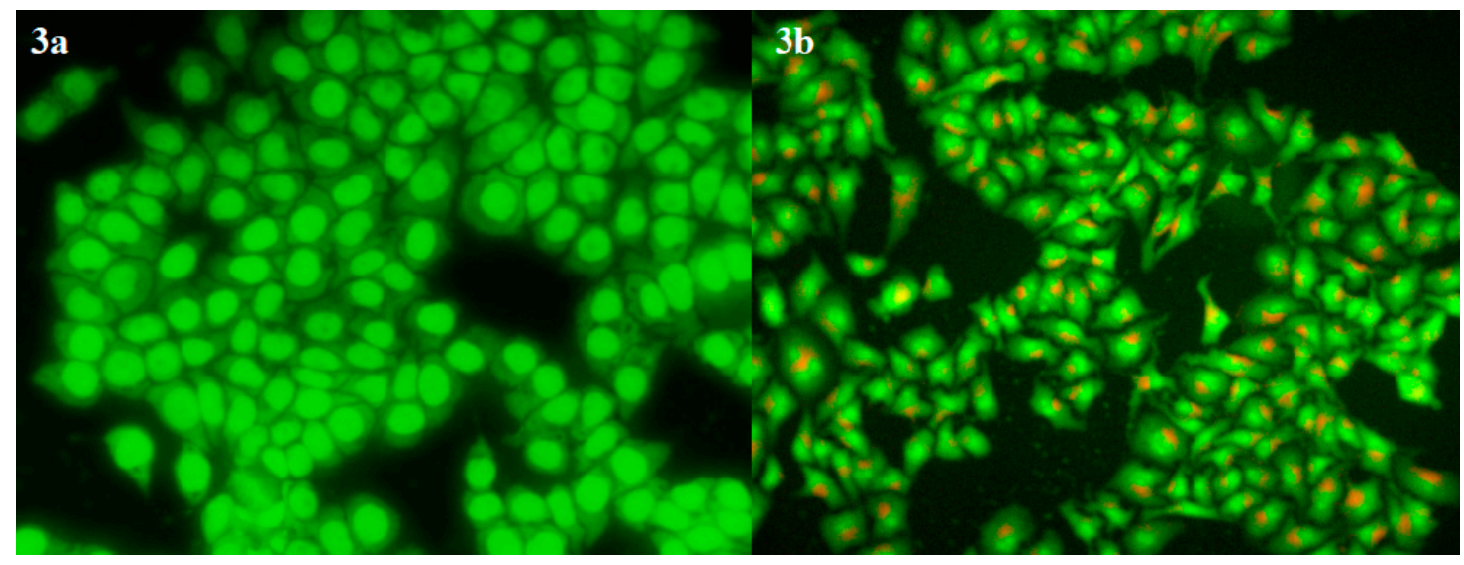

Figure 3. The cell morphology of HepG2 cells of the control and test groups. (a) HepG2 cells treated without any compounds; (b) HepG2 cells were treated with $0.01 \mu \mathrm{M}$ for $12 \mathrm{~h}$; Changes of nuclear morphology were determined by AO staining.

\subsection{Molecular Docking Study}

In order to explore the binding mode of the target compounds with the c-Met active site, molecular docking simulation studies were performed using AutoDock vina v1.02 (The Scripps Research Institute, La Jolla, CA, USA) and Discovery Studio 3.5 (Dassault Systèmes BIOVIA, San Diego, CA, USA). Based on the in vitro inhibition results, we selected compound 53, our best c-Met inhibitor in this study, as the ligand example, and the structure of c-Met was selected as the docking model (PDB ID code: 3LQ8).

The binding modes of compound 53 and c-Met were depicted in Figure $4 \mathrm{a}, \mathrm{b}$. In the binding mode, compound 53 is potently bound to the active binding site of c-Met via four hydrogen bonds and one pi-pi interactions. The the quinoline group (A ring) formed one hydrogen bond and one pi-pi interaction with MET 1160 and TYR 1159 respectively. Then the oxygen atom of the 5-atom linker moiety and the nitrogen atom of the pyridazinone moiety ( $\mathrm{C}$ ring) formed bidentate hydrogen bond with the amino hydrogen of ASP 1222. In addition, the oxygen atom of the pyridazinone moiety ( $\mathrm{C}$ ring) formed one hydrogen bond with the amino hydrogen of LYS 1110 . In general, 
these results of the molecular docking study showed that 6,7-disubstituted-4-phenoxyquinoline derivatives bearing pyridazinone moiety could act synergistically to interact with the active binding site of c-Met, suggested that compound 53 may be a potential inhibitor of c-Met.
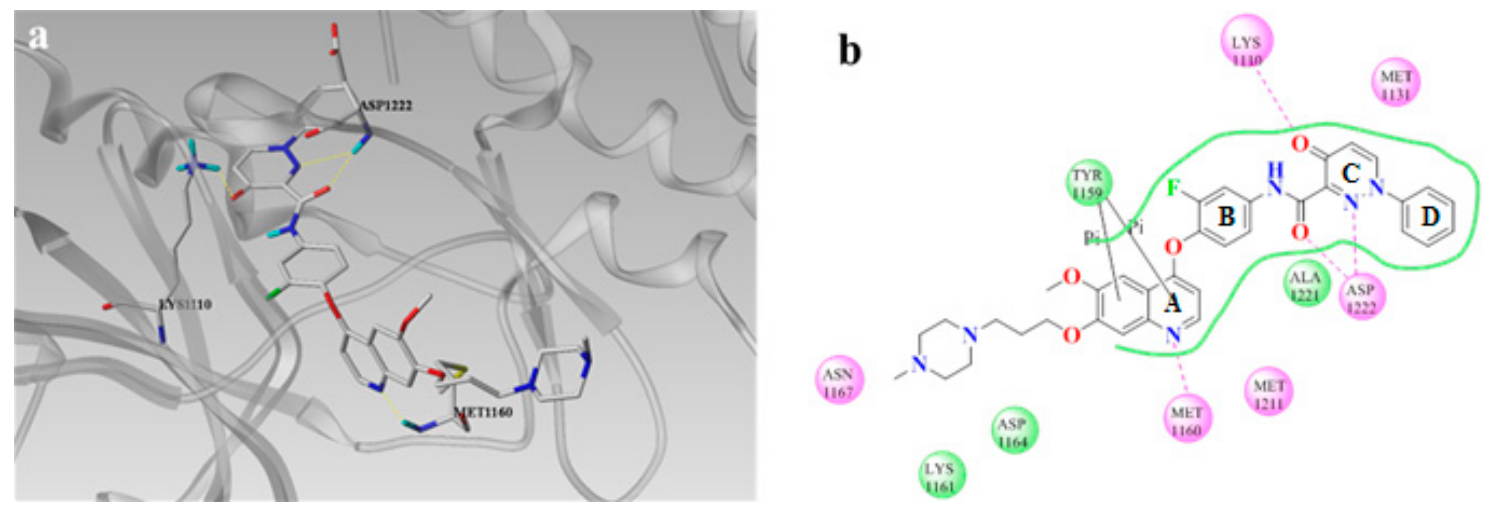

Figure 4. The docking mode of $\mathbf{5 3}$ with c-Met. (a) The 3D interaction map between the $\mathbf{5 3}$ and c-Met, the hydrogen bonds was colored with yellow; (b) 2D depiction of the ligand-protein interaction.

\section{Experimental Section}

\subsection{General Information}

All melting points were obtained on a Büchi Melting Point B-540 apparatus (Büchi Labortechnik, Flawil, Switzerland) and were uncorrected. Nuclear Magnetic Resonance (NMR) spectra were performed using Bruker $400 \mathrm{MHz}$ spectrometers (Bruker Bioscience, Billerica, MA, USA) with tetramethylsilane (TMS) as an internal standard. Mass spectra (MS) were taken in electrospray ionization (ESI) mode on Agilent 1100 Liquid chromatography-mass spectrometry (LC-MS) (Agilent, Palo Alto, CA, USA). Thin layer chromatography (TLC) analysis was carried out on silica gel plates GF254 (Qingdao Haiyang Chemical, Qingdao, China). All materials were obtained from commercial suppliers and used without purification, unless otherwise specified. Yields were optimized.

\subsection{Chemistry}

\subsubsection{Preparation of 3-Fluoro-4-(6,7-disubstituted quinolin-4-yloxy)anilines 15a-d}

The preparation of the key intermediates $15 a-d$ has been illustrated in detail in our previous work [22], and so the synthesis method would not be listed here.

\subsubsection{Preparation of Compounds 17a-h}

Appropriate substituted anilines $\mathbf{1 6 a}-\mathbf{h}(0.101 \mathrm{~mol})$ was added to the mixture solution of hydrogen chloride/water $(1: 1,10 \mathrm{~mL})$, sodium nitrite $(0.1 \mathrm{~mol})$ and stirred for $5 \mathrm{~min}$ at $0{ }^{\circ} \mathrm{C}$, respectively. Then the mixture was added to the ethanol solution which ammonium acetate $(1 \mathrm{~mol})$ and ethyl acetoacetate $(0.1 \mathrm{~mol})$ were dissolved in. The reaction was monitored by TLC until completed. Finally the solution was filtered and washed with a plenty of water to give a yellow solid.

\subsubsection{Preparation of Compounds 18a-h}

The yellow solid 17a-h $(0.115 \mathrm{~mol})$ was dissolve in the DMF-DMA $(50 \mathrm{~mL})$, heated from $0{ }^{\circ} \mathrm{C}$ to $100{ }^{\circ} \mathrm{C}$ and stirred for $3-4 \mathrm{~h}$. After the reaction was completed, poured the mixture to the petroleum ether and filtered to obtain the compounds 18a-h. 


\subsubsection{Preparation of Compounds $19 \mathbf{a}-\mathbf{h}$}

The compounds $\mathbf{1 8 a}-\mathbf{h}$ were dissolved in ethanol at $80{ }^{\circ} \mathrm{C}$. Then $10 \% \mathrm{NaOH}$ was added in the reaction solution slowly, refluxed for $1 \mathrm{~h}$ and monitored by TLC. The solution was concentrated in vacuum, the residue was resolved with dichloromethane $(300 \mathrm{~mL})$, washed with brine $(60 \mathrm{~mL})$, dried over anhydrous $\mathrm{Na}_{2} \mathrm{SO}_{4}$, and concentrated in vacuum to give the yellow solids 19a-h.

\subsubsection{Preparation of Compounds $\mathbf{2 0 a}-\mathbf{h}$}

Ethanol $(100 \mathrm{~mL})$ and water $(10 \mathrm{~mL})$ were added to a flask containing compounds $19 \mathbf{a}-\mathbf{h}(0.01 \mathrm{~mol})$, and then sodium hydroxide $(0.02 \mathrm{~mol})$ was added portionwise, and the reaction was carried out at room temperature. As the reaction progresses, a large amount of precipitate is generated. Water was added to dissolve the precipitate, and then activated carbon was added to remove the pigment and remove it from the aqueous solution. The filtrate was extracted and the $\mathrm{pH}$ adjusted to 3-4 with hydrochloric acid to give a white solid $\mathbf{2 0 a}-\mathbf{h}$.

\subsubsection{Preparation of Compounds 21a-h}

The compounds $\mathbf{2 0 a}-\mathbf{h}(1 \mathrm{mmol})$ and appropriate DMF $(0.1 \mathrm{mmol})$ were dissolved in dichloromethane, then appropriate oxalyl chloride was added slowly and monitored by TLC. The solution was used for next step without further purification.

\subsubsection{Preparation of Compounds 22-53}

A solution of phenylpyrdazinone carbonyl chloride $\mathbf{2 1 a}-\mathbf{h}(0.82 \mathrm{mmol})$ in dichloromethane $(10 \mathrm{~mL})$ was added drop-wise to a solution of aniline $15 \mathbf{a}-\mathbf{d}(0.41 \mathrm{mmol})$ and diisopropylethylamine $(0.49 \mathrm{mmol})$ in dichloromethane $(10 \mathrm{~mL})$ in an ice bath. Upon completion of the addition, the reaction mixture was removed from the ice bath and placed in room temperature for $30 \mathrm{~min}$ and monitored by TLC. The mixture was concentrated in vacuum to yield 22-53 which were recrystallized by isopropanol.

N-(3-Fluoro-4-((6-methoxy-7-(3-morpholinopropoxy)quinolin-4-yl)xy)phenyl)-1-4-fluorophenyl)-4-oxo-1,4dihydropyridazine-3-carboxamide (22). Light yellow solid. $37.2 \%$ yield, m.p: $152.3-153.5{ }^{\circ} \mathrm{C} .{ }^{1} \mathrm{H}$ NMR (400 MHz, DMSO) $\delta 12.02(\mathrm{~s}, 1 \mathrm{H}), 8.99(\mathrm{~d}, J=7.8 \mathrm{~Hz}, 1 \mathrm{H}), 8.48(\mathrm{~d}, J=5.2 \mathrm{~Hz}, 1 \mathrm{H}), 8.02(\mathrm{~d}, J=12.7 \mathrm{~Hz}$, $1 \mathrm{H}), 7.86(\mathrm{dd}, J=8.9,4.6 \mathrm{~Hz}, 2 \mathrm{H}), 7.55(\mathrm{~d}, J=12.2 \mathrm{~Hz}, 2 \mathrm{H}), 7.49(\mathrm{dt}, J=15.2,7.6 \mathrm{~Hz}, 3 \mathrm{H}), 7.40(\mathrm{~s}, 1 \mathrm{H})$, $6.93(\mathrm{~d}, J=7.8 \mathrm{~Hz}, 1 \mathrm{H}), 6.49(\mathrm{~d}, J=5.2 \mathrm{~Hz}, 1 \mathrm{H}), 4.20(\mathrm{t}, J=6.4 \mathrm{~Hz}, 2 \mathrm{H}), 3.95(\mathrm{~s}, 3 \mathrm{H}), 3.59(\mathrm{t}, J=4.2 \mathrm{~Hz}$, $4 \mathrm{H}), 2.47(\mathrm{~d}, J=7.0 \mathrm{~Hz}, 2 \mathrm{H}), 2.40(\mathrm{~s}, 4 \mathrm{H}), 2.02-1.94(\mathrm{~m}, 2 \mathrm{H}) .{ }^{13} \mathrm{C}$ NMR $(101 \mathrm{MHz}, \mathrm{DMSO}) \delta 169.09$, $162.83,159.92,159.17,154.74,152.30,151.93,149.58,148.81,147.50,146.37,142.03,139.41,137.05,136.95$, $124.33,123.87,123.79,120.46,116.62,116.38,114.45,108.53,102.09,101.50,99.02,66.66,66.18(2 C)$, 55.76, 54.79, 53.34(2C), 25.66. TOF MS ES+ $(\mathrm{m} / z):(\mathrm{M}+\mathrm{H})^{+}$, calcd for $\mathrm{C}_{34} \mathrm{H}_{31} \mathrm{~F}_{2} \mathrm{~N}_{5} \mathrm{O}_{6}: 644.2321$, found, 644.2304 .

1-(4-Chlorophenyl)-N-(3-fluoro-4-((6-methoxy-7-(3-morpholinopropoxy)quinolin-4-yl)oxy)phenyl)-4-oxo-1,4dihydropyridazine-3-carboxamide (23). Light yellow solid. 36.2\% yield, m.p: $138.2-139.1{ }^{\circ} \mathrm{C} .{ }^{1} \mathrm{H}$ NMR (400 MHz, DMSO) $\delta 11.95(\mathrm{~s}, 1 \mathrm{H}), 9.02(\mathrm{~d}, J=7.9 \mathrm{~Hz}, 1 \mathrm{H}), 8.49(\mathrm{~d}, J=5.0 \mathrm{~Hz}, 1 \mathrm{H}), 8.02(\mathrm{~d}, J=12.8 \mathrm{~Hz}$, $1 \mathrm{H}), 7.86(\mathrm{~d}, J=8.5 \mathrm{~Hz}, 2 \mathrm{H}), 7.70(\mathrm{~d}, J=8.5 \mathrm{~Hz}, 2 \mathrm{H}), 7.55(\mathrm{~d}, J=10.6 \mathrm{~Hz}, 2 \mathrm{H}), 7.50(\mathrm{t}, J=8.7 \mathrm{~Hz}, 1 \mathrm{H})$, $7.44(\mathrm{~s}, 1 \mathrm{H}), 6.93(\mathrm{~d}, J=7.8 \mathrm{~Hz}, 1 \mathrm{H}), 6.51(\mathrm{~d}, J=5.0 \mathrm{~Hz}, 1 \mathrm{H}), 4.26(\mathrm{~s}, 2 \mathrm{H}), 3.96(\mathrm{~s}, 3 \mathrm{H}), 3.78(\mathrm{~s}, 4 \mathrm{H})$, $3.04(\mathrm{~s}, 4 \mathrm{H}), 2.50-2.48(\mathrm{~m}, 2 \mathrm{H}), 2.20(\mathrm{~s}, 2 \mathrm{H})$. TOF MS ES+ $(\mathrm{m} / \mathrm{z}):(\mathrm{M}+\mathrm{H})^{+}$, calcd for $\mathrm{C}_{34} \mathrm{H}_{31} \mathrm{ClFN}_{5} \mathrm{O}_{6}$ : 660.2025 , found, 660.2009 .

1-(4-Bromophenyl)-N-(3-fluoro-4-((6-methoxy-7-(3-morpholinopropoxy)quinolin-4-yl)oxy)phenyl)-4-oxo-1,4dihydropyridazine-3-carboxamide (24). Light yellow solid. 37.9\% yield, m.p: 125.7-127.1 ${ }^{\circ} \mathrm{C} .{ }^{1} \mathrm{H}$ NMR (400 MHz, DMSO) $\delta 12.03(\mathrm{~s}, 1 \mathrm{H}), 9.12(\mathrm{~d}, J=7.8 \mathrm{~Hz}, 1 \mathrm{H}), 8.58(\mathrm{~d}, J=5.2 \mathrm{~Hz}, 1 \mathrm{H}), 8.11(\mathrm{~d}, J=12.8 \mathrm{~Hz}$, $1 \mathrm{H}), 7.91(\mathrm{q}, J=8.6 \mathrm{~Hz}, 4 \mathrm{H}), 7.66(\mathrm{~d}, J=9.9 \mathrm{~Hz}, 2 \mathrm{H}), 7.60(\mathrm{t}, J=8.7 \mathrm{~Hz}, 2 \mathrm{H}), 7.52(\mathrm{~s}, 1 \mathrm{H}), 7.03(\mathrm{~d}, J=7.8 \mathrm{~Hz}$, $1 \mathrm{H}), 6.60(\mathrm{~d}, J=5.1 \mathrm{~Hz}, 1 \mathrm{H}), 4.31(\mathrm{~d}, J=5.9 \mathrm{~Hz}, 2 \mathrm{H}), 4.05(\mathrm{~s}, 3 \mathrm{H}), 3.76(\mathrm{~s}, 4 \mathrm{H}), 2.76(\mathrm{~s}, 4 \mathrm{H}), 2.60(\mathrm{~s}, 2 \mathrm{H})$, $2.15(\mathrm{~s}, 2 \mathrm{H})$. TOF MS ES+ $(\mathrm{m} / z):(\mathrm{M}+\mathrm{H})^{+}$, calcd for $\mathrm{C}_{34} \mathrm{H}_{31} \mathrm{BrFN}_{5} \mathrm{O}_{6}$ : 704.1520, found, 704.1501 . 
N-(3-Fluoro-4-((6-methoxy-7-(3-morpholinopropoxy)quinolin-4-yl)oxy)phenyl)-1-(4-methoxyphenyl)-4-oxo-1,4dihydropyridazine-3-carboxamide (25). White solid. $38.1 \%$ yield, m.p: $153.8-155.2{ }^{\circ} \mathrm{C} .{ }^{1} \mathrm{H} \mathrm{NMR}(400 \mathrm{MHz}$, DMSO) $\delta 12.18(\mathrm{~s}, 1 \mathrm{H}), 8.95(\mathrm{~d}, J=7.8 \mathrm{~Hz}, 1 \mathrm{H}), 8.69(\mathrm{~d}, J=5.8 \mathrm{~Hz}, 1 \mathrm{H}), 8.08(\mathrm{~d}, J=12.6 \mathrm{~Hz}, 1 \mathrm{H})$, 7.77-7.67 (m, 3H), $7.59(\mathrm{dd}, J=20.1,9.7 \mathrm{~Hz}, 3 \mathrm{H}), 7.15(\mathrm{~d}, J=9.0 \mathrm{~Hz}, 2 \mathrm{H}), 6.92(\mathrm{~d}, J=7.8 \mathrm{~Hz}, 1 \mathrm{H})$, $6.81(\mathrm{~d}, J=4.7 \mathrm{~Hz}, 1 \mathrm{H}), 4.33(\mathrm{~s}, 2 \mathrm{H}), 4.02(\mathrm{~s}, 3 \mathrm{H}), 3.84(\mathrm{~s}, 3 \mathrm{H}), 3.49(\mathrm{~s}, 4 \mathrm{H}), 3.12(\mathrm{~s}, 4 \mathrm{H}), 2.50-2.49(\mathrm{~m}, 2 \mathrm{H})$, 2.35 (s, 2H). ${ }^{13} \mathrm{C}$ NMR (101 MHz, DMSO) $\delta 169.48,160.52,159.82,159.74,155.18,152.73,152.11,149.98$, $149.21,147.60,146.46,142.42,137.63,136.81,124.77,123.44(2 \mathrm{C}), 121.10,117.15,115.14(2 \mathrm{C}), 109.25$, $109.04,102.70,101.95,99.60,66.58,63.96,56.30,56.10(2 \mathrm{C}), 54.20,51.79(2 \mathrm{C}), 23.59$. TOF MS ES+ $(\mathrm{m} / z)$ : $(\mathrm{M}+\mathrm{H})^{+}$, calcd for $\mathrm{C}_{35} \mathrm{H}_{34} \mathrm{FN}_{5} \mathrm{O}_{7}: 656.2521$, found, 656.2503.

1-(3-Chloro-4-fluorophenyl)-N-(3-fluoro-4-((6-methoxy-7-(3-morpholinopropoxy)quinolin-4-yl)oxy)phenyl)4-oxo-1,4-dihydropyridazine-3-carboxamide (26). Pure white solid. 35.1\% yield, m.p: 138.1-140.6 ${ }^{\circ} \mathrm{C}$. ${ }^{1} \mathrm{H}$ NMR (400 MHz, DMSO) $\delta 11.84(\mathrm{~s}, 1 \mathrm{H}), 8.93(\mathrm{~d}, J=7.3 \mathrm{~Hz}, 1 \mathrm{H}), 8.39$ (d, J = 4.6 Hz, 1H), 8.05 (s, $\left.1 \mathrm{H}\right)$, $7.92(\mathrm{~d}, J=12.9 \mathrm{~Hz}, 1 \mathrm{H}), 7.77(\mathrm{~s}, 1 \mathrm{H}), 7.61(\mathrm{t}, J=8.8 \mathrm{~Hz}, 1 \mathrm{H}), 7.47(\mathrm{~d}, J=11.6 \mathrm{~Hz}, 2 \mathrm{H}), 7.44-7.38(\mathrm{~m}, 1 \mathrm{H})$, $7.31(\mathrm{~s}, 1 \mathrm{H}), 6.85(\mathrm{~d}, J=7.5 \mathrm{~Hz}, 1 \mathrm{H}), 6.40(\mathrm{~d}, J=4.8 \mathrm{~Hz}, 1 \mathrm{H}), 4.11(\mathrm{~s}, 2 \mathrm{H}), 3.87(\mathrm{~s}, 3 \mathrm{H}), 3.50(\mathrm{~s}, 4 \mathrm{H})$, $2.37(\mathrm{~d}, J=6.5 \mathrm{~Hz}, 2 \mathrm{H}), 2.31(\mathrm{~s}, 4 \mathrm{H}), 1.90(\mathrm{~d}, J=5.5 \mathrm{~Hz}, 2 \mathrm{H}) .{ }^{13} \mathrm{C}$ NMR $(101 \mathrm{MHz}, \mathrm{DMSO}) \delta 169.67$, $160.19,159.65,158.56,156.09,155.23,152.78,152.45,150.11,149.26,147.94,146.91,142.28,140.13,137.45$, $124.72,124.21,122.72,120.84,118.39,118.17,117.17,115.01,109.09,102.62,99.57,67.19,66.70(2 C)$, 56.26, 55.29, 53.85(2C), 26.19. TOF MS ES+ $(m / z):(\mathrm{M}+\mathrm{H})^{+}$, calcd for $\mathrm{C}_{34} \mathrm{H}_{30} \mathrm{ClF}_{2} \mathrm{~N}_{5} \mathrm{O}_{6}: 678.1931$, found, 678.1908 .

1-(4-Bromo-2-fluorophenyl)-N-(3-fluoro-4-((6-methoxy-7-(3-morpholinopropoxy)quinolin-4-yl)oxy)phenyl)4-oxo-1,4-dihydropyridazine-3-carboxamide (27). White solid. $35.7 \%$ yield, m.p: $113.7-115.1{ }^{\circ} \mathrm{C}$. ${ }^{1} \mathrm{H}$ NMR (400 MHz, DMSO) $\delta 11.82(\mathrm{~s}, 1 \mathrm{H}), 8.75(\mathrm{~d}, J=7.7 \mathrm{~Hz}, 1 \mathrm{H}), 8.47(\mathrm{~d}, J=5.2 \mathrm{~Hz}, 1 \mathrm{H}), 7.97(\mathrm{~d}, J=10.3 \mathrm{~Hz}$, 2H), $7.76(\mathrm{t}, J=8.3 \mathrm{~Hz}, 1 \mathrm{H}), 7.69(\mathrm{~d}, J=8.7 \mathrm{~Hz}, 1 \mathrm{H}), 7.53(\mathrm{~s}, 2 \mathrm{H}), 7.51-7.45(\mathrm{~m}, 1 \mathrm{H}), 7.40(\mathrm{~s}, 1 \mathrm{H})$, $6.90(\mathrm{~d}, J=7.8 \mathrm{~Hz}, 1 \mathrm{H}), 6.48(\mathrm{~d}, J=5.0 \mathrm{~Hz}, 1 \mathrm{H}), 4.19(\mathrm{t}, J=6.1 \mathrm{~Hz}, 2 \mathrm{H}), 3.95(\mathrm{~s}, 3 \mathrm{H}), 3.59(\mathrm{~d}, J=3.7 \mathrm{~Hz}$, $4 \mathrm{H}), 2.46(\mathrm{t}, J=6.9 \mathrm{~Hz}, 2 \mathrm{H}), 2.39(\mathrm{~s}, 4 \mathrm{H}), 2.04-1.93(\mathrm{~m}, 2 \mathrm{H})$. TOF MS ES+ $(\mathrm{m} / \mathrm{z}):(\mathrm{M}+\mathrm{H})^{+}$, calcd for $\mathrm{C}_{34} \mathrm{H}_{30} \mathrm{BrF}_{2} \mathrm{~N}_{5} \mathrm{O}_{6}:$ 722.1426, found, 722.1403 .

1-(4-Chloro-3-(trifluoromethyl)phenyl)-N-(3-fluoro-4-((6-methoxy-7-(3-morpholinopropoxy)quinolin-4-yl)oxy) phenyl)-4-oxo-1,4-dihydropyridazine-3-carboxamide (28). Light yellow solid. 47.3\% yield, m.p: 203.5-204.7 ${ }^{\circ} \mathrm{C} .{ }^{1} \mathrm{H}$ NMR (400 MHz, DMSO) $\delta 11.75(\mathrm{~s}, 1 \mathrm{H}), 9.02(\mathrm{~d}, J=7.8 \mathrm{~Hz}, 1 \mathrm{H})$, $8.37(\mathrm{~d}, J=5.3 \mathrm{~Hz}, 1 \mathrm{H}), 8.19(\mathrm{~s}, 1 \mathrm{H}), 8.06(\mathrm{~d}, J=7.1 \mathrm{~Hz}, 1 \mathrm{H}), 7.91(\mathrm{~d}, J=9.6 \mathrm{~Hz}, 2 \mathrm{H}), 7.49-7.42(\mathrm{~m}$, $2 \mathrm{H}), 7.39(\mathrm{~d}, J=9.3 \mathrm{~Hz}, 1 \mathrm{H}), 7.30(\mathrm{~s}, 1 \mathrm{H}), 6.85(\mathrm{~d}, J=7.9 \mathrm{~Hz}, 1 \mathrm{H}), 6.39(\mathrm{~d}, J=5.2 \mathrm{~Hz}, 1 \mathrm{H}), 4.10(\mathrm{~s}, 2 \mathrm{H})$, $3.85(\mathrm{~s}, 3 \mathrm{H}), 3.49(\mathrm{~s}, 4 \mathrm{H}), 2.38-2.37(\mathrm{~m}, 2 \mathrm{H}), 2.30(\mathrm{~s}, 4 \mathrm{H}), 1.88(\mathrm{~s}, 2 \mathrm{H}) .{ }^{13} \mathrm{C} \mathrm{NMR}(101 \mathrm{MHz}, \mathrm{DMSO})$ $\delta 169.17,159.80,159.18,154.74,152.28,151.90,149.60,148.83,148.05,146.35,141.80,141.62,136.87,136.41$, 133.06, 130.37, 126.69, 124.31, 120.91, 120.86, 120.19, 116.70, 114.52, 108.83, 108.60, 102.17, 99.07, 66.61, 65.84(2C), 55.80, 54.67, 53.09(2C), 25.32. TOF MS ES+ $(m / z):(\mathrm{M}+\mathrm{H})^{+}$, calcd for $\mathrm{C}_{35} \mathrm{H}_{30} \mathrm{ClF}_{4} \mathrm{~N}_{5} \mathrm{O}_{6}$ : 728.1899 , found, 728.1875 .

N-(3-Fluoro-4-((6-methoxy-7-(3-morpholinopropoxy)quinolin-4-yl)oxy)phenyl)-4-oxo-1-phenyl-1,4-dihydrop yridazine-3-carboxamide (29). Pure white solid. 39.7\% yield, m.p: $118.7-119.8{ }^{\circ} \mathrm{C} .{ }^{1} \mathrm{H} \mathrm{NMR}(400 \mathrm{MHz}$, DMSO) $\delta 12.00(\mathrm{~s}, 1 \mathrm{H}), 9.03(\mathrm{~d}, J=7.8 \mathrm{~Hz}, 1 \mathrm{H}), 8.47(\mathrm{~d}, J=5.2 \mathrm{~Hz}, 1 \mathrm{H}), 8.02(\mathrm{~d}, J=12.7 \mathrm{~Hz}, 1 \mathrm{H})$, $7.82(\mathrm{~d}, J=7.7 \mathrm{~Hz}, 2 \mathrm{H}), 7.63(\mathrm{t}, J=7.2 \mathrm{~Hz}, 2 \mathrm{H}), 7.59-7.47(\mathrm{~m}, 4 \mathrm{H}), 7.40(\mathrm{~s}, 1 \mathrm{H}), 6.93(\mathrm{~d}, J=7.9 \mathrm{~Hz}$, $1 \mathrm{H}), 6.49(\mathrm{~d}, J=5.3 \mathrm{~Hz}, 1 \mathrm{H}), 4.20(\mathrm{t}, J=6.1 \mathrm{~Hz}, 2 \mathrm{H}), 3.95(\mathrm{~s}, 3 \mathrm{H}), 3.59(\mathrm{~s}, 4 \mathrm{H}), 2.50-2.48(\mathrm{~m}, 2 \mathrm{H})$, $2.40(\mathrm{~s}, 4 \mathrm{H}), 1.99(\mathrm{~d}, \mathrm{~J}=6.6 \mathrm{~Hz}, 2 \mathrm{H})$. TOF MS ES+ $(\mathrm{m} / z):(\mathrm{M}+\mathrm{H})^{+}$, calcd for $\mathrm{C}_{34} \mathrm{H}_{32} \mathrm{FN}_{5} \mathrm{O}_{6}: 626.2415$, found, 626.2396 .

N-(3-Fluoro-4-((6-methoxy-7-(3-(piperidin-1-yl)propoxy)quinolin-4-yl)oxy)phenyl)-1-(4-fluorophenyl)-4-oxo -1,4-dihydropyridazine-3-carboxamide (30). Light yellow solid. 43.4\% yield, m.p: $149.4-151.1{ }^{\circ} \mathrm{C}$. ${ }^{1} \mathrm{H}$ NMR (400 MHz, DMSO) $\delta 12.03(\mathrm{~s}, 1 \mathrm{H}), 9.00(\mathrm{~d}, J=7.8 \mathrm{~Hz}, 1 \mathrm{H}), 8.48(\mathrm{~d}, J=5.1 \mathrm{~Hz}, 1 \mathrm{H}), 8.03(\mathrm{~d}, J=12.5 \mathrm{~Hz}$, $1 \mathrm{H}), 7.87(\mathrm{dd}, J=8.6,4.3 \mathrm{~Hz}, 2 \mathrm{H}), 7.56(\mathrm{~d}, J=10.6 \mathrm{~Hz}, 2 \mathrm{H}), 7.50(\mathrm{dd}, J=16.0,7.8 \mathrm{~Hz}, 3 \mathrm{H}), 7.41(\mathrm{~s}, 1 \mathrm{H})$, $6.94(\mathrm{~d}, J=7.8 \mathrm{~Hz}, 1 \mathrm{H}), 6.50(\mathrm{~d}, J=5.0 \mathrm{~Hz}, 1 \mathrm{H}), 4.20(\mathrm{~d}, J=6.1 \mathrm{~Hz}, 2 \mathrm{H}), 3.96(\mathrm{~s}, 3 \mathrm{H}), 2.51(\mathrm{~s}, 2 \mathrm{H})$, 
$2.42(\mathrm{~s}, 4 \mathrm{H}), 2.00(\mathrm{~d}, J=6.7 \mathrm{~Hz}, 2 \mathrm{H}), 1.53(\mathrm{~s}, 4 \mathrm{H}), 1.41(\mathrm{~s}, 2 \mathrm{H}) .{ }^{13} \mathrm{C}$ NMR (101 MHz, DMSO) $\delta$ 169.09, $162.82,160.37,159.92,159.17,154.74,152.29,151.93,149.59,148.80,147.49,146.38,142.02,139.40$, $124.31,123.87,123.78,120.46,116.62(2 \mathrm{C}), 116.38,114.46,108.77,108.53,102.09,99.02,66.77,55.76,54.98$, 53.97(2C), 25.91, 25.39(2C), 23.94. TOF MS ES+ $(m / z):(\mathrm{M}+\mathrm{H})^{+}$, calcd for $\mathrm{C}_{35} \mathrm{H}_{33} \mathrm{~F}_{2} \mathrm{~N}_{5} \mathrm{O}_{5}: 642.2528$, found, 642.2511 .

1-(4-Chlorophenyl)-N-(3-fluoro-4-((6-methoxy-7-(3-(piperidin-1-yl)propoxy)quinolin-4-yl)oxy)phenyl)-4-oxo -1,4-dihydropyridazine-3-carboxamide (31). White solid. 42.7\% yield, m.p: $158.2-159.6{ }^{\circ} \mathrm{C} .{ }^{1} \mathrm{H}$ NMR (400 MHz, DMSO) $\delta 12.05(\mathrm{~s}, 1 \mathrm{H}), 9.11(\mathrm{~d}, J=7.8 \mathrm{~Hz}, 1 \mathrm{H}), 8.57(\mathrm{~d}, J=5.1 \mathrm{~Hz}, 1 \mathrm{H}), 8.11(\mathrm{~d}, J=12.4 \mathrm{~Hz}$, $1 \mathrm{H}), 7.95(\mathrm{~d}, J=8.6 \mathrm{~Hz}, 2 \mathrm{H}), 7.80(\mathrm{~d}, J=8.6 \mathrm{~Hz}, 2 \mathrm{H}), 7.70-7.55(\mathrm{~m}, 3 \mathrm{H}), 7.49(\mathrm{~s}, 1 \mathrm{H}), 7.03(\mathrm{~d}, J=7.8 \mathrm{~Hz}$, $1 \mathrm{H}), 6.58(\mathrm{~d}, J=4.9 \mathrm{~Hz}, 1 \mathrm{H}), 4.27(\mathrm{~d}, J=6.0 \mathrm{~Hz}, 2 \mathrm{H}), 4.05(\mathrm{~s}, 3 \mathrm{H}), 2.52(\mathrm{t}, J=6.8 \mathrm{~Hz}, 2 \mathrm{H}), 2.44(\mathrm{~s}, 4 \mathrm{H})$, $2.06(\mathrm{~d}, J=6.0 \mathrm{~Hz}, 2 \mathrm{H}), 1.60(\mathrm{~s}, 4 \mathrm{H}), 1.48(\mathrm{~s}, 2 \mathrm{H}) .{ }^{13} \mathrm{C}$ NMR (101 MHz, DMSO) $\delta 169.65,160.56,159.75$, 155.31, 152.57, 150.20, 149.38, 148.49, 146.95, 142.19, 142.17, 142.16, 137.63, 133.58, 130.20(2C), 124.87, 123.61(2C), 120.95, 117.25, 115.04, 109.35, 109.12, 102.69, 99.63, 67.44, 56.36, 55.65, 54.67(2C), 26.68, 26.17(2C), 24.70. TOF MS ES+ $(\mathrm{m} / \mathrm{z})$ : $(\mathrm{M}+\mathrm{H})^{+}$, calcd for $\mathrm{C}_{35} \mathrm{H}_{33} \mathrm{ClFN}_{5} \mathrm{O}_{5}$ : 658.2233, found, 658.2209.

1-(4-Bromophenyl)-N-(3-fluoro-4-((6-methoxy-7-(3-(piperidin-1-yl)propoxy)quinolin-4-yl)oxy)phenyl)-4-oxo -1,4-dihydropyridazine-3-carboxamide (32). Light yellow solid. $48.3 \%$ yield, m.p: $141.3-143.4{ }^{\circ} \mathrm{C} .{ }^{1} \mathrm{H}$ NMR (400 MHz, DMSO) $\delta 11.94(\mathrm{~s}, 1 \mathrm{H}), 9.02(\mathrm{~d}, J=7.8 \mathrm{~Hz}, 1 \mathrm{H}), 8.49(\mathrm{~d}, J=5.2 \mathrm{~Hz}, 1 \mathrm{H}), 8.02(\mathrm{~d}, J=12.7 \mathrm{~Hz}$, $1 \mathrm{H}), 7.84(\mathrm{~d}, J=9.0 \mathrm{~Hz}, 2 \mathrm{H}), 7.79(\mathrm{~d}, J=8.5 \mathrm{~Hz}, 2 \mathrm{H}), 7.56(\mathrm{~s}, 2 \mathrm{H}), 7.50(\mathrm{t}, J=8.8 \mathrm{~Hz}, 1 \mathrm{H}), 7.43(\mathrm{~s}, 1 \mathrm{H})$, $6.93(\mathrm{~d}, J=7.9 \mathrm{~Hz}, 1 \mathrm{H}), 6.51(\mathrm{~d}, J=5.0 \mathrm{~Hz}, 1 \mathrm{H}), 4.23(\mathrm{~s}, 2 \mathrm{H}), 3.96(\mathrm{~s}, 3 \mathrm{H}), 2.80(\mathrm{~s}, 4 \mathrm{H}), 2.12(\mathrm{~s}, 2 \mathrm{H})$, $1.64(\mathrm{~s}, 4 \mathrm{H}), 1.47(\mathrm{~s}, 2 \mathrm{H}), 1.23(\mathrm{~s}, 2 \mathrm{H})$. TOF MS ES+ $(\mathrm{m} / \mathrm{z}):(\mathrm{M}+\mathrm{H})^{+}$, calcd for $\mathrm{C}_{35} \mathrm{H}_{33} \mathrm{BrFN}_{5} \mathrm{O}_{5}: 702.1727$, found, 702.1703 .

N-(3-Fluoro-4-((6-methoxy-7-(3-(piperidin-1-yl)propoxy)quinolin-4-yl)oxy)phenyl)-1-(4-methoxyphenyl)-4oxo-1,4-dihydropyridazine-3-carboxamide (33). White solid. $35.2 \%$ yield, m.p: $105.4-106 .{ }^{\circ} \mathrm{C} .{ }^{1} \mathrm{H}$ NMR $(400 \mathrm{MHz}, \mathrm{DMSO}) \delta 12.14(\mathrm{~s}, 1 \mathrm{H}), 8.94(\mathrm{~d}, J=7.7 \mathrm{~Hz}, 1 \mathrm{H}), 8.47(\mathrm{~d}, J=4.9 \mathrm{~Hz}, 1 \mathrm{H}), 8.02(\mathrm{~d}, J=12.7 \mathrm{~Hz}$, $1 \mathrm{H}), 7.72(\mathrm{~d}, J=7.7 \mathrm{~Hz}, 2 \mathrm{H}), 7.55(\mathrm{~d}, J=8.8 \mathrm{~Hz}, 2 \mathrm{H}), 7.49(\mathrm{t}, J=8.8 \mathrm{~Hz}, 1 \mathrm{H}), 7.40(\mathrm{~s}, 1 \mathrm{H}), 7.15(\mathrm{~d}, J=7.7 \mathrm{~Hz}$, $2 \mathrm{H}), 6.92(\mathrm{~d}, J=7.7 \mathrm{~Hz}, 1 \mathrm{H}), 6.49(\mathrm{~d}, J=4.9 \mathrm{~Hz}, 1 \mathrm{H}), 4.19(\mathrm{~s}, 2 \mathrm{H}), 3.95(\mathrm{~s}, 3 \mathrm{H}), 3.83(\mathrm{~s}, 3 \mathrm{H}), 2.50(\mathrm{~s}, 4 \mathrm{H})$, 2.49-2.40 (m, 2H), $2.01(\mathrm{~d}, J=6.1 \mathrm{~Hz}, 2 \mathrm{H}), 1.54(\mathrm{~s}, 4 \mathrm{H}), 1.41(\mathrm{~s}, 2 \mathrm{H}) .{ }^{13} \mathrm{C}$ NMR (101 MHz, DMSO) $\delta$ 169.05, 159.97, 159.28, 159.18, 154.73, 152.29, 151.88, 149.57, 148.81, 147.00, 146.35, 141.92, 136.98, 136.36, 124.30, 122.95(2C), 120.66, 116.65, 114.68(2C), 114.49, 108.76, 108.55, 102.10, 99.04, 66.69, 55.77, $55.62,54.83,53.76(2 \mathrm{C}), 25.61,25.07(2 \mathrm{C}), 23.65$. TOF MS ES+ $(\mathrm{m} / z):(\mathrm{M}+\mathrm{H})^{+}$, calcd for $\mathrm{C}_{36} \mathrm{H}_{36} \mathrm{FN}_{5} \mathrm{O}_{6}$ : 654.2728 , found, 654.2705 .

1-(3-Chloro-4-fluorophenyl)-N-(3-fluoro-4-((6-methoxy-7-(3-(piperidin-1-yl)propoxy)quinolin-4-yl)oxy)phen yl)-4-oxo-1,4-dihydropyridazine-3-carboxamide (34). Light yellow solid. $37.9 \%$ yield, m.p: $121.7-123.4{ }^{\circ} \mathrm{C}$. ${ }^{1} \mathrm{H}$ NMR (400 MHz, DMSO) $\delta 11.93(\mathrm{~s}, 1 \mathrm{H}), 9.02(\mathrm{~d}, J=7.8 \mathrm{~Hz}, 1 \mathrm{H}), 8.48(\mathrm{~d}, J=5.2 \mathrm{~Hz}, 1 \mathrm{H}), 8.17-8.13$ $(\mathrm{m}, 1 \mathrm{H}), 8.03(\mathrm{~d}, J=13.0 \mathrm{~Hz}, 1 \mathrm{H}), 7.90-7.84(\mathrm{~m}, 1 \mathrm{H}), 7.71(\mathrm{t}, J=9.0 \mathrm{~Hz}, 1 \mathrm{H}), 7.60-7.54(\mathrm{~m}, 2 \mathrm{H})$, $7.51(\mathrm{t}, J=8.7 \mathrm{~Hz}, 1 \mathrm{H}), 7.40(\mathrm{~s}, 1 \mathrm{H}), 6.94(\mathrm{~d}, J=7.9 \mathrm{~Hz}, 1 \mathrm{H}), 6.50(\mathrm{~d}, J=5.2 \mathrm{~Hz}, 1 \mathrm{H}), 4.19(\mathrm{t}, J=6.3 \mathrm{~Hz}$, $2 \mathrm{H}), 3.96(\mathrm{~s}, 3 \mathrm{H}), 2.43(\mathrm{t}, J=7.0 \mathrm{~Hz}, 2 \mathrm{H}), 2.36(\mathrm{~s}, 4 \mathrm{H}), 2.00-1.93(\mathrm{~m}, 2 \mathrm{H}), 1.55-1.47(\mathrm{~m}, 4 \mathrm{H}), 1.39(\mathrm{~s}, 2 \mathrm{H})$. TOF MS ES+ $(m / z):(\mathrm{M}+\mathrm{H})^{+}$, calcd for $\mathrm{C}_{35} \mathrm{H}_{32} \mathrm{ClF}_{2} \mathrm{~N}_{5} \mathrm{O}_{5}: 676.2138$, found, 676.2109.

1-(4-Bromo-2-fluorophenyl)-N-(3-fluoro-4-((6-methoxy-7-(3-(piperidin-1-yl)propoxy)quinolin-4-yl)oxy)phen yl)-4-oxo-1,4-dihydropyridazine-3-carboxamide (35). White solid. $42.1 \%$ yield, m.p: $105.9-107.4{ }^{\circ} \mathrm{C}$. ${ }^{1} \mathrm{H}$ NMR (400 MHz, DMSO) $\delta 11.79(\mathrm{~s}, 1 \mathrm{H}), 8.74(\mathrm{~d}, J=7.9 \mathrm{~Hz}, 1 \mathrm{H}), 8.45(\mathrm{~d}, J=5.1 \mathrm{~Hz}, 1 \mathrm{H})$, $7.96(\mathrm{~d}, J=11.9 \mathrm{~Hz}, 2 \mathrm{H}), 7.74(\mathrm{t}, J=8.3 \mathrm{~Hz}, 1 \mathrm{H}), 7.69(\mathrm{~d}, J=8.9 \mathrm{~Hz}, 1 \mathrm{H}), 7.57-7.50(\mathrm{~m}, 2 \mathrm{H})$, $7.48(\mathrm{t}, J=8.8 \mathrm{~Hz}, 1 \mathrm{H}), 7.38(\mathrm{~s}, 1 \mathrm{H}), 6.90(\mathrm{~d}, J=7.9 \mathrm{~Hz}, 1 \mathrm{H}), 6.47(\mathrm{~d}, J=5.2 \mathrm{~Hz}, 1 \mathrm{H}), 4.17(\mathrm{t}, J=6.3 \mathrm{~Hz}$, $2 \mathrm{H}), 3.93(\mathrm{~s}, 3 \mathrm{H}), 2.48(\mathrm{~s}, 4 \mathrm{H}), 2.41(\mathrm{t}, J=7.0 \mathrm{~Hz}, 2 \mathrm{H}), 1.99-1.89(\mathrm{~m}, 2 \mathrm{H}), 1.55-1.42(\mathrm{~m}, 4 \mathrm{H}), 1.36(\mathrm{~s}, 2 \mathrm{H})$. ${ }^{13} \mathrm{C}$ NMR (101 MHz, DMSO) $\delta$ 168.74, 159.81, 159.16, 154.73, 152.27, 151.96, 149.60, 149.03, 148.80, 146.37, 144.52, 136.87, 130.47, 128.70, 128.53, 124.33, 122.89, 120.60, 120.38, 119.69, 116.64, 114.45, 108.75, $108.51,102.07,99.02,66.81,55.77,55.05,54.06(2 \mathrm{C}), 26.04,25.54(2 \mathrm{C}), 24.08$. TOF MS ES+ $(\mathrm{m} / \mathrm{z}):(\mathrm{M}+\mathrm{H})^{+}$, calcd for $\mathrm{C}_{35} \mathrm{H}_{32} \mathrm{BrF}_{2} \mathrm{~N}_{5} \mathrm{O}_{5}: 720.1633$, found, 720.1612 . 
1-(4-Chloro-3-(trifluoromethyl)phenyl)-N-(3-fluoro-4-((6-methoxy-7-(3-(piperidin-1-yl)propoxy)quinolin-4yl)oxy)phenyl)-4-oxo-1,4-dihydropyridazine-3-carboxamide (36). Light yellow solid. 48.7\% yield, m.p: 185.7-186.6 ${ }^{\circ} \mathrm{C} .{ }^{1} \mathrm{H}$ NMR (400 MHz, DMSO) $\delta 11.77(\mathrm{~s}, 1 \mathrm{H}), 9.01(\mathrm{~d}, J=8.0 \mathrm{~Hz}, 1 \mathrm{H})$, $8.37(\mathrm{~d}, J=5.1 \mathrm{~Hz}, 1 \mathrm{H}), 8.19(\mathrm{~s}, 1 \mathrm{H}), 8.06(\mathrm{~d}, J=8.8 \mathrm{~Hz}, 1 \mathrm{H}), 7.90(\mathrm{~d}, J=8.6 \mathrm{~Hz}, 2 \mathrm{H}), 7.46(\mathrm{~d}, J=13.6 \mathrm{~Hz}$, $2 \mathrm{H}), 7.39(\mathrm{t}, J=8.8 \mathrm{~Hz}, 1 \mathrm{H}), 7.30(\mathrm{~s}, 1 \mathrm{H}), 6.84(\mathrm{~d}, J=7.8 \mathrm{~Hz}, 1 \mathrm{H}), 6.39(\mathrm{~d}, J=5.0 \mathrm{~Hz}, 1 \mathrm{H}), 4.10(\mathrm{~s}, 2 \mathrm{H})$, $3.85(\mathrm{~s}, 3 \mathrm{H}), 2.56(\mathrm{~s}, 4 \mathrm{H}), 1.95(\mathrm{~s}, 2 \mathrm{H}), 1.48(\mathrm{~s}, 4 \mathrm{H}), 1.34(\mathrm{~s}, 2 \mathrm{H}), 1.12(\mathrm{~s}, 2 \mathrm{H})$. TOF MS ES+ $(\mathrm{m} / \mathrm{z}):(\mathrm{M}+\mathrm{H})^{+}$, calcd for $\mathrm{C}_{36} \mathrm{H}_{32} \mathrm{ClF}_{4} \mathrm{~N}_{5} \mathrm{O}_{5}: 726.2106$, found, 726.2097 .

N-(3-Fluoro-4-((6-methoxy-7-(3-(piperidin-1-yl)propoxy)quinolin-4-yl)oxy)phenyl)-4-oxo-1-phenyl-1,4-dihy dropyridazine-3-carboxamide (37). Pure white solid. 38.9\% yield, m.p: $152.3-153.1^{\circ} \mathrm{C} .{ }^{1} \mathrm{H}$ NMR $(400 \mathrm{MHz}$, DMSO) $\delta 12.02(\mathrm{~s}, 1 \mathrm{H}), 9.03(\mathrm{~d}, J=7.7 \mathrm{~Hz}, 1 \mathrm{H}), 8.47(\mathrm{~d}, J=4.6 \mathrm{~Hz}, 1 \mathrm{H}), 8.02(\mathrm{~d}, J=12.7 \mathrm{~Hz}, 1 \mathrm{H})$, $7.81(\mathrm{~d}, J=7.6 \mathrm{~Hz}, 2 \mathrm{H}), 7.63(\mathrm{t}, J=7.3 \mathrm{~Hz}, 2 \mathrm{H}), 7.58-7.46(\mathrm{~m}, 4 \mathrm{H}), 7.39(\mathrm{~s}, 1 \mathrm{H}), 6.94(\mathrm{~d}, J=7.7 \mathrm{~Hz}, 1 \mathrm{H})$, $6.49(\mathrm{~d}, J=4.5 \mathrm{~Hz}, 1 \mathrm{H}), 4.18(\mathrm{~s}, 2 \mathrm{H}), 3.95(\mathrm{~s}, 3 \mathrm{H}), 2.43(\mathrm{t}, J=6.7 \mathrm{~Hz}, 2 \mathrm{H}), 2.35(\mathrm{~s}, 4 \mathrm{H}), 1.96(\mathrm{~d}, J=6.0 \mathrm{~Hz}$, 2H), $1.50(\mathrm{~s}, 4 \mathrm{H}), 1.39(\mathrm{~s}, 2 \mathrm{H}) .{ }^{13} \mathrm{C}$ NMR (101 MHz, DMSO) $\delta 168.65,159.48,158.66,154.24,151.79$, 151.46, 149.10, 148.28, 147.12, 145.89, 142.34, 141.26, 136.56, 129.20(2C), 128.11, 123.79, 120.82(2C), $120.02,116.14,113.97,108.27,108.04,101.60,98.54,66.32,55.27,54.53,53.54(2 \mathrm{C}), 25.54,25.02(2 \mathrm{C}), 23.56$. TOF MS ES+ $(\mathrm{m} / z):(\mathrm{M}+\mathrm{H})^{+}$, calcd for $\mathrm{C}_{35} \mathrm{H}_{34} \mathrm{FN}_{5} \mathrm{O}_{5}: 624.2622$, found, 624.2605.

$\mathrm{N}$-(3-Fluoro-4-((6-methoxy-7-(3-(4-methylpiperidin-1-yl)propoxy)quinolin-4-yl)oxy)phenyl)-1-(4-fluorophen yl)-4-oxo-1,4-dihydropyridazine-3-carboxamide (38). White solid. $35.8 \%$ yield, m.p: $210.1-211.4{ }^{\circ} \mathrm{C}$. ${ }^{1} \mathrm{H}$ NMR $(400 \mathrm{MHz}, \mathrm{DMSO}) \delta 12.02(\mathrm{~s}, 1 \mathrm{H}), 9.00(\mathrm{~d}, J=7.8 \mathrm{~Hz}, 1 \mathrm{H}), 8.49(\mathrm{~s}, 1 \mathrm{H}), 8.03(\mathrm{~d}, J=12.9 \mathrm{~Hz}$, $1 \mathrm{H}), 7.88(\mathrm{~d}, J=4.8 \mathrm{~Hz}, 2 \mathrm{H}), 7.64-7.45(\mathrm{~m}, 5 \mathrm{H}), 7.40(\mathrm{~s}, 1 \mathrm{H}), 6.94(\mathrm{~d}, J=7.4 \mathrm{~Hz}, 1 \mathrm{H}), 6.50(\mathrm{~s}, 1 \mathrm{H})$, $4.19(\mathrm{~s}, 2 \mathrm{H}), 3.96(\mathrm{~s}, 3 \mathrm{H}), 2.87(\mathrm{~d}, J=10.5 \mathrm{~Hz}, 2 \mathrm{H}), 2.46(\mathrm{~s}, 2 \mathrm{H}), 1.97(\mathrm{~s}, 2 \mathrm{H}), 1.91(\mathrm{~d}, J=10.2 \mathrm{~Hz}, 2 \mathrm{H})$, $1.59(\mathrm{~d}, J=11.5 \mathrm{~Hz}, 2 \mathrm{H}), 1.33(\mathrm{~s}, 1 \mathrm{H}), 1.15(\mathrm{~d}, J=12.2 \mathrm{~Hz}, 2 \mathrm{H}), 0.89(\mathrm{~d}, J=6.0 \mathrm{~Hz}, 3 \mathrm{H})$. TOF MS ES+ $(\mathrm{m} / \mathrm{z})$ : $(\mathrm{M}+\mathrm{H})^{+}$, calcd for $\mathrm{C}_{36} \mathrm{H}_{35} \mathrm{~F}_{2} \mathrm{~N}_{5} \mathrm{O}_{5}$ : 656.2685, found, 656.2673.

1-(4-Chlorophenyl)-N-(3-fluoro-4-((6-methoxy-7-(3-(4-methylpiperidin-1-yl)propoxy)quinolin-4-yl)oxy)phen yl)-4-oxo-1,4-dihydropyridazine-3-carboxamide (39). Light yellow solid. $51.4 \%$ yield, m.p: $220.6-222.1^{\circ} \mathrm{C}$. ${ }^{1} \mathrm{H}$ NMR $(400 \mathrm{MHz}, \mathrm{DMSO}) \delta 11.96(\mathrm{~s}, 1 \mathrm{H}), 9.02(\mathrm{~d}, J=7.8 \mathrm{~Hz}, 1 \mathrm{H}), 8.48(\mathrm{~d}, J=4.4 \mathrm{~Hz}, 1 \mathrm{H})$, $8.02(\mathrm{~d}, J=12.9 \mathrm{~Hz}, 1 \mathrm{H}), 7.86(\mathrm{~d}, J=7.5 \mathrm{~Hz}, 2 \mathrm{H}), 7.70(\mathrm{~d}, J=7.5 \mathrm{~Hz}, 2 \mathrm{H}), 7.59-7.46(\mathrm{~m}, 3 \mathrm{H}), 7.39(\mathrm{~s}, 1 \mathrm{H})$, $6.94(\mathrm{~d}, J=7.9 \mathrm{~Hz}, 1 \mathrm{H}), 6.49(\mathrm{~d}, J=4.6 \mathrm{~Hz}, 1 \mathrm{H}), 4.18(\mathrm{~s}, 2 \mathrm{H}), 3.96(\mathrm{~s}, 3 \mathrm{H}), 2.85(\mathrm{~d}, J=10.3 \mathrm{~Hz}, 2 \mathrm{H})$, $2.44(\mathrm{t}, J=6.4 \mathrm{~Hz}, 2 \mathrm{H}), 1.97(\mathrm{~d}, J=6.2 \mathrm{~Hz}, 2 \mathrm{H}), 1.88(\mathrm{t}, J=11.2 \mathrm{~Hz}, 2 \mathrm{H}), 1.57(\mathrm{~d}, J=11.7 \mathrm{~Hz}, 2 \mathrm{H})$, $1.30(\mathrm{~s}, 1 \mathrm{H}), 1.16(\mathrm{t}, J=11.5 \mathrm{~Hz}, 2 \mathrm{H}), 0.89(\mathrm{~d}, J=6.0 \mathrm{~Hz}, 3 \mathrm{H}) .{ }^{13} \mathrm{C}$ NMR $(101 \mathrm{MHz}, \mathrm{DMSO}) \delta 169.67$, $160.51,159.74,155.31,152.87,152.57,150.22,149.38,148.42,146.98,142.18,137.59,136.96,133.59$, 130.21(2C), 124.87, 123.60(2C), 120.97, 117.24, 115.06, 109.36, 109.14, 102.71, 99.66, 67.43, 56.37, 55.25, 54.02(2C), 34.57(2C), 30.93, 26.79, 22.34. TOF MS ES+ $(\mathrm{m} / z)$ : $(\mathrm{M}+\mathrm{H})^{+}$, calcd for $\mathrm{C}_{36} \mathrm{H}_{35} \mathrm{ClFN}_{5} \mathrm{O}_{5}$ : 672.2389 , found, 672.2367 .

1-(4-Bromophenyl)-N-(3-fluoro-4-((6-methoxy-7-(3-(4-methylpiperidin-1-yl)propoxy)quinolin-4-yl)oxy)phen yl)-4-oxo-1,4-dihydropyridazine-3-carboxamide (40). Light yellow solid. $49.8 \%$ yield, m.p: $220.7-221.4{ }^{\circ} \mathrm{C}$. ${ }^{1} \mathrm{H}$ NMR (400 MHz, DMSO) $\delta 11.94(\mathrm{~s}, 1 \mathrm{H}), 9.03(\mathrm{~d}, J=7.4 \mathrm{~Hz}, 1 \mathrm{H}), 8.49(\mathrm{~s}, 1 \mathrm{H}), 8.02(\mathrm{~d}, J=12.3 \mathrm{~Hz}, 1 \mathrm{H})$, $7.84(\mathrm{~d}, J=8.7 \mathrm{~Hz}, 2 \mathrm{H}), 7.80(\mathrm{~d}, J=8.0 \mathrm{~Hz}, 2 \mathrm{H}), 7.55(\mathrm{~s}, 2 \mathrm{H}), 7.50(\mathrm{~s}, 1 \mathrm{H}), 7.40(\mathrm{~s}, 1 \mathrm{H}), 6.94(\mathrm{~d}, J=7.8 \mathrm{~Hz}$, $1 \mathrm{H}), 6.51(\mathrm{~s}, 1 \mathrm{H}), 4.20(\mathrm{~s}, 2 \mathrm{H}), 3.96(\mathrm{~s}, 3 \mathrm{H}), 2.92(\mathrm{~s}, 2 \mathrm{H}), 2.53(\mathrm{~s}, 2 \mathrm{H}), 2.00(\mathrm{~s}, 4 \mathrm{H}), 1.61(\mathrm{~d}, J=11.7 \mathrm{~Hz}, 2 \mathrm{H})$, $1.36(\mathrm{~s}, 1 \mathrm{H}), 1.17(\mathrm{~d}, J=12.0 \mathrm{~Hz}, 2 \mathrm{H}), 0.90(\mathrm{~d}, J=5.8 \mathrm{~Hz}, 3 \mathrm{H})$. TOF MS ES+ $(\mathrm{m} / z):(\mathrm{M}+\mathrm{H})^{+}$, calcd for $\mathrm{C}_{36} \mathrm{H}_{35} \mathrm{BrFN}_{5} \mathrm{O}_{5}$ : 716.1884, found, 716.1863 .

$\mathrm{N}$-(3-Fluoro-4-((6-methoxy-7-(3-(4-methylpiperidin-1-yl)propoxy)quinolin-4-yl)oxy)phenyl)-1-(4-methoxyph enyl)-4-oxo-1,4-dihydropyridazine-3-carboxamide (41). Light yellow solid. $40.3 \%$ yield, m.p: $145.1-146.3^{\circ} \mathrm{C}$. ${ }^{1} \mathrm{H}$ NMR $(400 \mathrm{MHz}, \mathrm{DMSO}) \delta 12.12(\mathrm{~s}, 1 \mathrm{H}), 8.94(\mathrm{~d}, J=7.7 \mathrm{~Hz}, 1 \mathrm{H}), 8.47(\mathrm{~d}, J=5.3 \mathrm{~Hz}, 1 \mathrm{H})$, $8.02(\mathrm{~d}, J=12.9 \mathrm{~Hz}, 1 \mathrm{H}), 7.73(\mathrm{~d}, J=8.7 \mathrm{~Hz}, 2 \mathrm{H}), 7.54(\mathrm{~s}, 2 \mathrm{H}), 7.49(\mathrm{t}, J=8.3 \mathrm{~Hz}, 1 \mathrm{H}), 7.39(\mathrm{~s}, 1 \mathrm{H})$, $7.16(\mathrm{~d}, J=7.4 \mathrm{~Hz}, 2 \mathrm{H}), 6.92(\mathrm{~d}, J=8.0 \mathrm{~Hz}, 1 \mathrm{H}), 6.50(\mathrm{~s}, 1 \mathrm{H}), 4.18(\mathrm{~s}, 2 \mathrm{H}), 3.95(\mathrm{~s}, 3 \mathrm{H}), 3.84(\mathrm{~s}, 3 \mathrm{H})$, $2.85(\mathrm{~d}, J=9.0 \mathrm{~Hz}, 2 \mathrm{H}), 2.44(\mathrm{~d}, J=6.5 \mathrm{~Hz}, 2 \mathrm{H}), 1.97(\mathrm{~d}, J=6.9 \mathrm{~Hz}, 2 \mathrm{H}), 1.87(\mathrm{t}, J=11.4 \mathrm{~Hz}, 2 \mathrm{H})$, 
$1.57(\mathrm{~d}, J=11.9 \mathrm{~Hz}, 2 \mathrm{H}), 1.30(\mathrm{~s}, 1 \mathrm{H}), 1.19-1.11(\mathrm{~m}, 2 \mathrm{H}), 0.88(\mathrm{~d}, J=6.3 \mathrm{~Hz}, 3 \mathrm{H})$. TOF MS ES+ $(\mathrm{m} / z)$ : $(\mathrm{M}+\mathrm{H})^{+}$, calcd for $\mathrm{C}_{37} \mathrm{H}_{38} \mathrm{FN}_{5} \mathrm{O}_{6}$ : 668.2884, found, 668.2836.

1-(3-Chloro-4-fluorophenyl)-N-(3-fluoro-4-((6-methoxy-7-(3-(4-methylpiperidin-1-yl)propoxy)quinolin-4-yl) oxy)phenyl)-4-oxo-1,4-dihydropyridazine-3-carboxamide (42). Light yellow solid. $41.5 \%$ yield, m.p: $\quad 112.5-113.7{ }^{\circ} \mathrm{C} .{ }^{1} \mathrm{H}$ NMR $(400 \mathrm{MHz}, \mathrm{DMSO}) \delta 11.94(\mathrm{~s}, 1 \mathrm{H}), 9.03(\mathrm{~d}, J=7.8 \mathrm{~Hz}, 1 \mathrm{H})$, $8.49(\mathrm{~d}, J=5.1 \mathrm{~Hz}, 1 \mathrm{H}), 8.15(\mathrm{~d}, J=4.0 \mathrm{~Hz}, 1 \mathrm{H}), 8.03(\mathrm{~d}, J=12.7 \mathrm{~Hz}, 1 \mathrm{H}), 7.88(\mathrm{~d}, J=9.0 \mathrm{~Hz}, 1 \mathrm{H})$, $7.71(\mathrm{t}, J=8.8 \mathrm{~Hz}, 1 \mathrm{H}), 7.57(\mathrm{~d}, J=13.1 \mathrm{~Hz}, 2 \mathrm{H}), 7.51(\mathrm{t}, J=8.8 \mathrm{~Hz}, 1 \mathrm{H}), 7.42(\mathrm{~s}, 1 \mathrm{H}), 6.94(\mathrm{~d}, J=7.8 \mathrm{~Hz}$, $1 \mathrm{H}), 6.50(\mathrm{~d}, J=5.0 \mathrm{~Hz}, 1 \mathrm{H}), 4.22(\mathrm{~s}, 2 \mathrm{H}), 3.97(\mathrm{~s}, 3 \mathrm{H}), 3.10(\mathrm{~s}, 2 \mathrm{H}), 2.75(\mathrm{~s}, 2 \mathrm{H}), 2.09(\mathrm{~s}, 2 \mathrm{H})$, $1.67(\mathrm{~d}, J=12.3 \mathrm{~Hz}, 2 \mathrm{H}), 1.30(\mathrm{~s}, 1 \mathrm{H}), 1.25(\mathrm{~d}, J=12.3 \mathrm{~Hz}, 4 \mathrm{H}), 0.91(\mathrm{~d}, J=6.3 \mathrm{~Hz}, 3 \mathrm{H})$. TOF MS ES+ $(m / z):(\mathrm{M}+\mathrm{H})^{+}$, calcd for $\mathrm{C}_{36} \mathrm{H}_{34} \mathrm{ClF}_{2} \mathrm{~N}_{5} \mathrm{O}_{5}: 690.2295$, found, 690.2273.

1-(4-Bromo-2-fluorophenyl)-N-(3-fluoro-4-((6-methoxy-7-(3-(4-methylpiperidin-1-yl)propoxy)quinolin-4-yl) oxy)phenyl)-4-oxo-1,4-dihydropyridazine-3-carboxamide (43). White solid. $35.3 \%$ yield, m.p: $115.8-117.3^{\circ} \mathrm{C}$. ${ }^{1} \mathrm{H}$ NMR (400 MHz, DMSO) $\delta 11.78(\mathrm{~s}, 1 \mathrm{H}), 8.74(\mathrm{~d}, J=7.9 \mathrm{~Hz}, 1 \mathrm{H}), 8.46(\mathrm{~s}, 1 \mathrm{H}), 7.96(\mathrm{~d}, J=12.2 \mathrm{~Hz}, 2 \mathrm{H})$, 7.79-7.66 (m, 2H), 7.52 (s, 2H), 7.51-7.44 (m, 1H), $7.37(\mathrm{~s}, 1 \mathrm{H}), 6.90(\mathrm{~d}, J=7.8 \mathrm{~Hz}, 1 \mathrm{H}), 6.47(\mathrm{~d}, J=5.1 \mathrm{~Hz}$, $1 \mathrm{H}), 4.17(\mathrm{t}, J=5.9 \mathrm{~Hz}, 2 \mathrm{H}), 3.93(\mathrm{~s}, 3 \mathrm{H}), 2.85(\mathrm{~d}, J=10.6 \mathrm{~Hz}, 2 \mathrm{H}), 2.45(\mathrm{~s}, 2 \mathrm{H}), 1.99-1.93(\mathrm{~m}, 2 \mathrm{H})$, $1.90(\mathrm{~d}, J=11.9 \mathrm{~Hz}, 2 \mathrm{H}), 1.56(\mathrm{~d}, J=12.3 \mathrm{~Hz}, 2 \mathrm{H}), 1.22(\mathrm{~d}, J=10.2 \mathrm{~Hz}, 1 \mathrm{H}), 1.13(\mathrm{~d}, J=12.3 \mathrm{~Hz}, 2 \mathrm{H})$, $0.87(\mathrm{~d}, J=6.3 \mathrm{~Hz}, 3 \mathrm{H}) .{ }^{13} \mathrm{C}$ NMR $(101 \mathrm{MHz}, \mathrm{DMSO}) \delta 168.76,159.78,159.16,154.73,153.28,151.96$, $149.60,148.97,148.78,146.38,136.87,130.37,128.71,128.70,128.52,124.32,122.97,122.89,120.61,120.38$, $119.71,116.64,108.76,108.52,102.08,99.03,66.80,55.76,54.67,53.43(2 C), 33.93(2 C), 30.33,26.16,21.77$. TOF MS ES+ $(m / z):(\mathrm{M}+\mathrm{H})^{+}$, calcd for $\mathrm{C}_{36} \mathrm{H}_{34} \mathrm{BrF}_{2} \mathrm{~N}_{5} \mathrm{O}_{5}: 734.1790$, found, 734.1773.

1-(4-Chloro-3-(trifluoromethyl)phenyl)-N-(3-fluoro-4-((6-methoxy-7-(3-(4-methylpiperidin-1-yl)propoxy)qui nolin-4-yl)oxy)phenyl)-4-oxo-1,4-dihydropyridazine-3-carboxamide (44). Light yellow solid. 38.7\% yield, m.p: 137.7-138.6 ${ }^{\circ} \mathrm{C} .{ }^{1} \mathrm{H}$ NMR (400 MHz, DMSO) $\delta 11.77$ (s, 1H), $9.02(\mathrm{~d}, J=7.9 \mathrm{~Hz}, 1 \mathrm{H})$, $8.37(\mathrm{~d}, J=5.3 \mathrm{~Hz}, 1 \mathrm{H}), 8.19(\mathrm{~s}, 1 \mathrm{H}), 8.06(\mathrm{~d}, J=8.4 \mathrm{~Hz}, 1 \mathrm{H}), 7.90(\mathrm{~d}, J=8.5 \mathrm{~Hz}, 2 \mathrm{H}), 7.50-7.42(\mathrm{~m}, 2 \mathrm{H})$, $7.39(\mathrm{t}, J=8.9 \mathrm{~Hz}, 1 \mathrm{H}), 7.30(\mathrm{~s}, 1 \mathrm{H}), 6.84(\mathrm{~d}, J=8.2 \mathrm{~Hz}, 1 \mathrm{H}), 6.39(\mathrm{~d}, J=5.3 \mathrm{~Hz}, 1 \mathrm{H}), 4.09(\mathrm{~s}, 2 \mathrm{H})$, $3.85(\mathrm{~s}, 3 \mathrm{H}), 2.91(\mathrm{~s}, 2 \mathrm{H}), 1.99(\mathrm{~d}, J=48.7 \mathrm{~Hz}, 4 \mathrm{H}), 1.53(\mathrm{~d}, J=10.0 \mathrm{~Hz}, 2 \mathrm{H}), 1.30(\mathrm{~s}, 1 \mathrm{H}), 1.12(\mathrm{~s}, 4 \mathrm{H})$, $0.79(\mathrm{~d}, J=6.3 \mathrm{~Hz}, 3 \mathrm{H}) .{ }^{13} \mathrm{C}$ NMR $(101 \mathrm{MHz}, \mathrm{DMSO}) \delta 169.66,160.26,159.66,155.22,152.77,152.34$, 150.03, 149.30, 148.44, 146.84, 142.26, 142.08, 137.47, 136.98, 133.53, 130.85, 127.13, 124.79, 121.39, 121.33, 120.70, 117.17, 115.00, 109.06, 102.61, 101.99, 99.53, 67.12, 56.25, 54.85, 53.52(2C), 33.67(2C), 30.28, 26.04, 22.04. TOF MS ES+ $(m / z):(\mathrm{M}+\mathrm{H})^{+}$, calcd for $\mathrm{C}_{37} \mathrm{H}_{34} \mathrm{ClF}_{4} \mathrm{~N}_{5} \mathrm{O}_{5}: 740.2263$, found, 740.2237.

N-(3-Fluoro-4-((6-methoxy-7-(3-(4-methylpiperidin-1-yl)propoxy)quinolin-4-yl)oxy)phenyl)-4-oxo-1-phenyl -1,4-dihydropyridazine-3-carboxamide (45). Pure white solid. 44.1\% yield, m.p: $148.5-150.1{ }^{\circ} \mathrm{C} .{ }^{1} \mathrm{H}$ NMR $(400 \mathrm{MHz}, \mathrm{DMSO}) \delta 12.02(\mathrm{~s}, 1 \mathrm{H}), 9.03(\mathrm{~d}, J=7.7 \mathrm{~Hz}, 1 \mathrm{H}), 8.47(\mathrm{~d}, J=5.1 \mathrm{~Hz}, 1 \mathrm{H}), 8.02(\mathrm{~d}, J=13.1 \mathrm{~Hz}$, $1 \mathrm{H}), 7.82(\mathrm{~d}, J=7.8 \mathrm{~Hz}, 2 \mathrm{H}), 7.63(\mathrm{t}, J=7.7 \mathrm{~Hz}, 2 \mathrm{H}), 7.51(\mathrm{dd}, J=17.2,8.4 \mathrm{~Hz}, 4 \mathrm{H}), 7.39(\mathrm{~s}, 1 \mathrm{H})$, $6.94(\mathrm{~d}, J=7.8 \mathrm{~Hz}, 1 \mathrm{H}), 6.49(\mathrm{~d}, J=4.8 \mathrm{~Hz}, 1 \mathrm{H}), 4.18(\mathrm{~s}, 2 \mathrm{H}), 3.95(\mathrm{~s}, 3 \mathrm{H}), 2.86(\mathrm{~d}, J=10.9 \mathrm{~Hz}, 2 \mathrm{H})$, $2.45(\mathrm{t}, J=6.7 \mathrm{~Hz}, 2 \mathrm{H}), 2.01-1.94(\mathrm{~m}, 2 \mathrm{H}), 1.89(\mathrm{t}, J=11.7 \mathrm{~Hz}, 2 \mathrm{H}), 1.57(\mathrm{~d}, J=11.2 \mathrm{~Hz}, 2 \mathrm{H}), 1.32(\mathrm{~s}, 1 \mathrm{H})$, $1.20-1.11(\mathrm{~m}, 2 \mathrm{H}), 0.88(\mathrm{~d}, J=6.2 \mathrm{~Hz}, 3 \mathrm{H}) .{ }^{13} \mathrm{C}$ NMR $(101 \mathrm{MHz}, \mathrm{DMSO}) \delta 169.63,160.54,159.68$, 155.25, 152.44, 150.13, 149.33, 148.26, 146.90, 143.37, 142.29, 137.48, 136.85, 130.23(2C), 129.13, 124.81, 121.86(2C), 121.00, 117.17, 115.02, 109.29, 109.12, 102.67, 99.60, 67.27, 56.31, 55.03, 53.76(2C), 34.12(2C), 30.58, 26.42, 22.16. TOF MS ES+ $(\mathrm{m} / z):(\mathrm{M}+\mathrm{H})^{+}$, calcd for $\mathrm{C}_{36} \mathrm{H}_{36} \mathrm{FN}_{5} \mathrm{O}_{5}: 638.2779$, found, 638.2754.

N-(3-Fluoro-4-((6-methoxy-7-(3-(4-methylpiperazin-1-yl)propoxy)quinolin-4-yl)oxy)phenyl)-1-(4-fluorophen yl)-4-oxo-1,4-dihydropyridazine-3-carboxamide (46). White solid. $36.1 \%$ yield, m.p: $184.6-185.7{ }^{\circ} \mathrm{C}$. ${ }^{1} \mathrm{H}$ NMR (400 MHz, DMSO) $\delta 12.03(\mathrm{~s}, 1 \mathrm{H}), 9.00(\mathrm{~d}, J=7.7 \mathrm{~Hz}, 1 \mathrm{H}), 8.48(\mathrm{~d}, J=4.7 \mathrm{~Hz}, 1 \mathrm{H})$, $8.02(\mathrm{~d}, J=12.5 \mathrm{~Hz}, 1 \mathrm{H}), 7.88(\mathrm{~s}, 2 \mathrm{H}), 7.55(\mathrm{~s}, 2 \mathrm{H}), 7.54-7.44(\mathrm{~m}, 3 \mathrm{H}), 7.40(\mathrm{~s}, 1 \mathrm{H}), 6.94(\mathrm{~d}, J=7.7 \mathrm{~Hz}, 1 \mathrm{H})$, $6.50(\mathrm{~s}, 1 \mathrm{H}), 4.19(\mathrm{~s}, 2 \mathrm{H}), 3.96(\mathrm{~s}, 3 \mathrm{H}), 2.47(\mathrm{~s}, 2 \mathrm{H}), 2.42(\mathrm{~d}, J=42.1 \mathrm{~Hz}, 8 \mathrm{H}), 2.17(\mathrm{~s}, 3 \mathrm{H}), 1.97(\mathrm{~s}, 2 \mathrm{H})$. ${ }^{13} \mathrm{C}$ NMR (101 MHz, DMSO) $\delta$ 168.57, 162.32, 159.87, 159.44, 158.67, 154.24, 151.44, 149.09, 148.31, $147.05,145.88,141.53,138.91,136.44,123.82,123.38(2 \mathrm{C}), 123.30,119.95,116.12(2 \mathrm{C}), 115.88,113.96$, 
108.03, 101.61, 98.53, 66.20, 55.27, 54.02(2C), 53.73, 51.91(2C), 44.90, 25.46. TOF MS ES+ $(\mathrm{m} / \mathrm{z}):(\mathrm{M}+\mathrm{H})^{+}$, calcd for $\mathrm{C}_{35} \mathrm{H}_{34} \mathrm{~F}_{2} \mathrm{~N}_{6} \mathrm{O}_{5}: 657.2637$, found, 657.2612.

1-(4-Chlorophenyl)-N-(3-fluoro-4-((6-methoxy-7-(3-(4-methylpiperazin-1-yl)propoxy)quinolin-4-yl)oxy)phe nyl)-4-oxo-1,4-dihydropyridazine-3-carboxamide (47). White solid. $59.1 \%$ yield, m.p: $163.5-165.3{ }^{\circ} \mathrm{C}$. ${ }^{1} \mathrm{H}$ NMR (400 MHz, DMSO) $\delta 11.95(\mathrm{~s}, 1 \mathrm{H}), 9.03(\mathrm{~d}, J=7.1 \mathrm{~Hz}, 1 \mathrm{H}), 8.48(\mathrm{~d}, J=4.5 \mathrm{~Hz}, 1 \mathrm{H})$, $8.02(\mathrm{~d}, J=13.1 \mathrm{~Hz}, 1 \mathrm{H}), 7.86(\mathrm{~d}, J=7.2 \mathrm{~Hz}, 2 \mathrm{H}), 7.71(\mathrm{~d}, J=8.3 \mathrm{~Hz}, 2 \mathrm{H}), 7.59-7.47(\mathrm{~m}, 3 \mathrm{H}), 7.40(\mathrm{~s}, 1 \mathrm{H})$, $6.94(\mathrm{~d}, J=7.8 \mathrm{~Hz}, 1 \mathrm{H}), 6.50(\mathrm{~s}, 1 \mathrm{H}), 4.19(\mathrm{~s}, 2 \mathrm{H}), 3.96(\mathrm{~s}, 3 \mathrm{H}), 2.46(\mathrm{~d}, J=6.6 \mathrm{~Hz}, 2 \mathrm{H}), 2.35-2.22(\mathrm{~s}, 8 \mathrm{H})$, $2.15(\mathrm{~s}, 3 \mathrm{H}), 1.97(\mathrm{~s}, 2 \mathrm{H})$. TOF MS ES+ $(\mathrm{m} / \mathrm{z}):(\mathrm{M}+\mathrm{H})^{+}$, calcd for $\mathrm{C}_{35} \mathrm{H}_{34} \mathrm{ClFN}_{6} \mathrm{O}_{5}:$ 673.2341, found, 673.2315 .

1-(4-Bromophenyl)-N-(3-fluoro-4-((6-methoxy-7-(3-(4-methylpiperazin-1-yl)propoxy)quinolin-4-yl)oxy)phen yl)-4-oxo-1,4-dihydropyridazine-3-carboxamide (48). White solid. $38.2 \%$ yield, m.p: $159.7-161.2{ }^{\circ} \mathrm{C}$. ${ }^{1} \mathrm{H}$ NMR (400 MHz, DMSO) $\delta 11.95(\mathrm{~s}, 1 \mathrm{H}), 9.03(\mathrm{~d}, J=7.9 \mathrm{~Hz}, 1 \mathrm{H}), 8.49(\mathrm{~d}, J=5.2 \mathrm{~Hz}, 1 \mathrm{H})$, $8.03(\mathrm{~d}, J=12.6 \mathrm{~Hz}, 1 \mathrm{H}), 7.82(\mathrm{~m}, J=8.9 \mathrm{~Hz}, 4 \mathrm{H}), 7.57(\mathrm{~d}, J=10.4 \mathrm{~Hz}, 2 \mathrm{H}), 7.54-7.48(\mathrm{~m}, 1 \mathrm{H})$, $7.42(\mathrm{~s}, 1 \mathrm{H}), 6.94(\mathrm{~d}, J=7.8 \mathrm{~Hz}, 1 \mathrm{H}), 6.51(\mathrm{~d}, J=5.1 \mathrm{~Hz}, 1 \mathrm{H}), 4.21(\mathrm{~s}, 2 \mathrm{H}), 3.97(\mathrm{~s}, 3 \mathrm{H}), 2.78(\mathrm{~s}, 4 \mathrm{H})$, $2.60(\mathrm{~s}, 4 \mathrm{H}), 2.47(\mathrm{~s}, 2 \mathrm{H}), 2.03(\mathrm{~d}, J=6.1 \mathrm{~Hz}, 2 \mathrm{H}), 1.23(\mathrm{~s}, 3 \mathrm{H})$. TOF MS ES+ $(\mathrm{m} / z):(\mathrm{M}+\mathrm{H})^{+}$, calcd for $\mathrm{C}_{35} \mathrm{H}_{34} \mathrm{BrFN}_{6} \mathrm{O}_{5}:$ 717.1836, found, 717.1804.

1-(4-((11-Oxidanyl)-15-methyl)phenyl)-N-(3-fluoro-4-((6-methoxy-7-(3-(4-methylpiperazin-1-yl)propoxy)qui nolin-4-yl)oxy)phenyl)-4-oxo-1,4-dihydropyridazine-3-carboxamide (49). Light yellow solid. $47.5 \%$ yield, m.p: $167.5-169.1{ }^{\circ} \mathrm{C} .{ }^{1} \mathrm{H}$ NMR (400 MHz, DMSO) $\delta 12.02(\mathrm{~s}, 1 \mathrm{H}), 8.83(\mathrm{~d}, J=7.7 \mathrm{~Hz}, 1 \mathrm{H})$, $8.36(\mathrm{~d}, J=4.0 \mathrm{~Hz}, 1 \mathrm{H}), 7.91(\mathrm{~d}, J=12.9 \mathrm{~Hz}, 1 \mathrm{H}), 7.62(\mathrm{~d}, J=8.8 \mathrm{~Hz}, 2 \mathrm{H}), 7.44(\mathrm{~d}, J=10.3 \mathrm{~Hz}, 2 \mathrm{H})$, $7.39(\mathrm{t}, J=8.7 \mathrm{~Hz}, 1 \mathrm{H}), 7.28(\mathrm{~s}, 1 \mathrm{H}), 7.05(\mathrm{~d}, J=8.7 \mathrm{~Hz}, 2 \mathrm{H}), 6.82(\mathrm{~d}, J=7.9 \mathrm{~Hz}, 1 \mathrm{H}), 6.39(\mathrm{~s}, 1 \mathrm{H})$, $4.08(\mathrm{~s}, 2 \mathrm{H}), 3.84(\mathrm{~s}, 3 \mathrm{H}), 3.73(\mathrm{~s}, 3 \mathrm{H}), 2.35(\mathrm{~d}, J=6.8 \mathrm{~Hz}, 2 \mathrm{H}), 2.37-2.14(\mathrm{~s}, 8 \mathrm{H}), 2.06(\mathrm{~s}, 3 \mathrm{H}), 1.86(\mathrm{~s}, 2 \mathrm{H})$. ${ }^{13} \mathrm{C}$ NMR (101 MHz, DMSO) $\delta$ 168.79, 159.80, 159.17, 155.77, 154.70, 153.23, 152.26, 151.91, 149.56, $148.93,148.78,146.33,144.46,137.06,128.67,128.49,124.27,122.96,120.57,120.34,119.70,116.66,114.45$, $108.46,102.05,99.00,66.65,66.18(3 \mathrm{C}), 55.74,54.79,53.34(3 \mathrm{C}), 25.65$. TOF MS ES+ $(\mathrm{m} / z):(\mathrm{M}+\mathrm{H})^{+}$, calcd for $\mathrm{C}_{36} \mathrm{H}_{37} \mathrm{FN}_{6} \mathrm{O}_{6}: 669.2837$, found, 669.2801.

1-(3-Chloro-4-fluorophenyl)-N-(3-fluoro-4-((6-methoxy-7-(3-(4-methylpiperazin-1-yl)propoxy)quinolin-4-yl) oxy)phenyl)-4-oxo-1,4-dihydropyridazine-3-carboxamide (50). Light yellow solid. $35.4 \%$ yield, m.p: 144.7-146.2 ${ }^{\circ} \mathrm{C} .{ }^{1} \mathrm{H}$ NMR (400 MHz, DMSO) $\delta 11.92(\mathrm{~s}, 1 \mathrm{H}), 9.02(\mathrm{~d}, J=7.9 \mathrm{~Hz}, 1 \mathrm{H})$, $8.48(\mathrm{~d}, J=5.1 \mathrm{~Hz}, 1 \mathrm{H}), 8.14(\mathrm{dd}, J=6.3,2.7 \mathrm{~Hz}, 1 \mathrm{H}), 8.02(\mathrm{~d}, J=12.6 \mathrm{~Hz}, 1 \mathrm{H}), 7.94-7.83(\mathrm{~m}, 1 \mathrm{H})$, $7.71(\mathrm{t}, J=9.0 \mathrm{~Hz}, 1 \mathrm{H}), 7.61-7.54(\mathrm{~m}, 2 \mathrm{H}), 7.51(\mathrm{t}, J=8.8 \mathrm{~Hz}, 1 \mathrm{H}), 7.40(\mathrm{~s}, 1 \mathrm{H}), 6.94(\mathrm{~d}, J=7.9 \mathrm{~Hz}$, $1 \mathrm{H}), 6.50(\mathrm{~d}, J=5.2 \mathrm{~Hz}, 1 \mathrm{H}), 4.19(\mathrm{t}, J=6.2 \mathrm{~Hz}, 2 \mathrm{H}), 3.96(\mathrm{~s}, 3 \mathrm{H}), 2.51(\mathrm{~s}, 2 \mathrm{H}), 2.49-2.45(\mathrm{~m}, 2 \mathrm{H})$, $2.35(\mathrm{~s}, 4 \mathrm{H}), 2.16(\mathrm{~s}, 3 \mathrm{H}), 2.02-1.93(\mathrm{~m}, 2 \mathrm{H}), 1.37-1.17(\mathrm{~m}, 2 \mathrm{H}) .{ }^{13} \mathrm{C}$ NMR (101 MHz, DMSO) $\delta 169.66$, 160.22, 159.66, 158.57, 156.09, 155.23, 152.47, 150.12, 149.27, 148.00, 146.88, 142.31, 140.17, 137.44, 137.03, 124.75, 124.24, 122.75, 120.84, 118.40, 118.18, 117.19, 115.00, 109.04, 102.62, 99.57, 67.26, 56.26, 55.24(2C), 54.82, 53.19(2C), 46.18, 26.54. TOF MS ES+ $(m / z):(\mathrm{M}+\mathrm{H})^{+}$, calcd for $\mathrm{C}_{35} \mathrm{H}_{33} \mathrm{ClF}_{2} \mathrm{~N}_{6} \mathrm{O}_{5}: 691.2247$, found, 691.2255 .

1-(4-Bromo-2-fluorophenyl)-N-(3-fluoro-4-((6-methoxy-7-(3-(4-methylpiperazin-1-yl)propoxy)quinolin-4-yl) oxy)phenyl)-4-oxo-1,4-dihydropyridazine-3-carboxamide (51). White solid. $39.8 \%$ yield, m.p: $103.1-104.7^{\circ} \mathrm{C}$. ${ }^{1} \mathrm{H}$ NMR (400 MHz, DMSO) $\delta 11.82(\mathrm{~s}, 1 \mathrm{H}), 8.76(\mathrm{dd}, J=7.8,1.8 \mathrm{~Hz}, 1 \mathrm{H}), 8.48(\mathrm{~d}, J=5.2 \mathrm{~Hz}, 1 \mathrm{H})$, $8.00(\mathrm{~s}, 1 \mathrm{H}), 7.97(\mathrm{~d}, J=1.9 \mathrm{~Hz}, 1 \mathrm{H}), 7.77(\mathrm{t}, J=8.4 \mathrm{~Hz}, 1 \mathrm{H}), 7.71(\mathrm{~d}, J=8.5 \mathrm{~Hz}, 1 \mathrm{H}), 7.55(\mathrm{~d}, J=8.8 \mathrm{~Hz}$, $2 \mathrm{H}), 7.49(\mathrm{t}, J=8.8 \mathrm{~Hz}, 1 \mathrm{H}), 7.40(\mathrm{~s}, 1 \mathrm{H}), 6.91(\mathrm{~d}, J=7.9 \mathrm{~Hz}, 1 \mathrm{H}), 6.49(\mathrm{~d}, J=5.1 \mathrm{~Hz}, 1 \mathrm{H}), 4.20(\mathrm{t}, J=6.4 \mathrm{~Hz}$, $2 \mathrm{H}), 3.96(\mathrm{~s}, 3 \mathrm{H}), 2.67(\mathrm{~s}, 4 \mathrm{H}), 2.57(\mathrm{~s}, 4 \mathrm{H}), 2.39(\mathrm{~s}, 3 \mathrm{H}), 2.05-1.95(\mathrm{~m}, 2 \mathrm{H}), 1.23(\mathrm{~s}, 2 \mathrm{H}) .{ }^{13} \mathrm{C} \mathrm{NMR}$ (101 MHz, DMSO) $\delta 169.21,160.34,159.67,156.32,155.21,153.78,152.76,152.43,150.10,149.60$, $149.32,146.85,144.99,129.19,129.05,124.79,123.46,121.10,120.87,120.16,117.16,114.99,109.04$, $102.62,99.56,67.16,56.29,54.55(3 \mathrm{C}), 52.32(2 \mathrm{C}), 45.29,26.33$. TOF MS ES+ $(\mathrm{m} / z)$ : $(\mathrm{M}+\mathrm{H})^{+}$, calcd for $\mathrm{C}_{35} \mathrm{H}_{33} \mathrm{BrF}_{2} \mathrm{~N}_{6} \mathrm{O}_{5}:$ 735.1742, found, 735.1713 . 
1-(4-Chloro-3-(trifluoromethyl)phenyl)-N-(3-fluoro-4-((6-methoxy-7-(3-(4-methylpiperazin-1-yl)propoxy)quin olin-4-yl)oxy)phenyl)-4-oxo-1,4-dihydropyridazine-3-carboxamide (52). Light yellow solid. 43.7\% yield, m.p: $211.7-212.6{ }^{\circ} \mathrm{C} .{ }^{1} \mathrm{H}$ NMR $(400 \mathrm{MHz}, \mathrm{DMSO}) \delta 11.77(\mathrm{~s}, 1 \mathrm{H}), 9.03(\mathrm{t}, J=8.1 \mathrm{~Hz}, 1 \mathrm{H}), 8.37(\mathrm{~d}, J=5.2 \mathrm{~Hz}$, $1 \mathrm{H}), 8.19(\mathrm{~s}, 1 \mathrm{H}), 8.06(\mathrm{~d}, J=8.7 \mathrm{~Hz}, 1 \mathrm{H}), 7.93-7.88(\mathrm{~m}, 2 \mathrm{H}), 7.48-7.42(\mathrm{~m}, 2 \mathrm{H}), 7.42-7.36(\mathrm{~m}, 1 \mathrm{H})$, $7.30(\mathrm{~d}, J=7.9 \mathrm{~Hz}, 1 \mathrm{H}), 6.85(\mathrm{t}, J=8.0 \mathrm{~Hz}, 1 \mathrm{H}), 6.38(\mathrm{~d}, J=5.2 \mathrm{~Hz}, 1 \mathrm{H}), 4.09(\mathrm{~d}, J=6.5 \mathrm{~Hz}, 2 \mathrm{H})$, $3.85(\mathrm{~d}, J=7.9 \mathrm{~Hz}, 3 \mathrm{H}), 2.42(\mathrm{~d}, J=6.3 \mathrm{~Hz}, 4 \mathrm{H}), 2.40(\mathrm{~s}, 4 \mathrm{H}), 2.20(\mathrm{~d}, J=7.6 \mathrm{~Hz}, 3 \mathrm{H}), 1.89(\mathrm{~d}, J=6.9 \mathrm{~Hz}$, 2H), 1.12 (s, 2H). ${ }^{13} \mathrm{C}$ NMR (101 MHz, DMSO) $\delta 169.16,159.80,159.17,154.73,152.28,151.94,149.61$, $148.80,148.03,146.38,141.60,139.90,136.99,136.53,133.04,132.81,130.34,126.64,125.45,124.27,120.86$, $120.19,116.70,114.51,108.58,102.14,99.08,66.67,55.78,54.02(3 C), 51.78(2 C), 44.76,25.85$. TOF MS $\mathrm{ES}+(\mathrm{m} / z):(\mathrm{M}+\mathrm{H})^{+}$, calcd for $\mathrm{C}_{36} \mathrm{H}_{33} \mathrm{ClF}_{4} \mathrm{~N}_{6} \mathrm{O}_{5}: 741.2215$, found, 741.2216.

N-(3-Fluoro-4-((6-methoxy-7-(3-(4-methylpiperazin-1-yl)propoxy)quinolin-4-yl)oxy)phenyl)-4-oxo-1-phenyl -1,4-dihydropyridazine-3-carboxamide (53). Pure white solid. 45.2\% yield, m.p: $151.3-152.1^{\circ} \mathrm{C} .{ }^{1} \mathrm{H}$ NMR (400 MHz, DMSO) $\delta 12.02(\mathrm{~s}, 1 \mathrm{H}), 9.03(\mathrm{~d}, J=7.8 \mathrm{~Hz}, 1 \mathrm{H}), 8.47(\mathrm{~d}, J=4.8 \mathrm{~Hz}, 1 \mathrm{H}), 8.02(\mathrm{~d}, J=12.0 \mathrm{~Hz}$, $1 \mathrm{H}), 7.82(\mathrm{~d}, J=7.4 \mathrm{~Hz}, 2 \mathrm{H}), 7.63(\mathrm{t}, J=7.4 \mathrm{~Hz}, 2 \mathrm{H}), 7.51(\mathrm{dd}, J=16.5,8.1 \mathrm{~Hz}, 4 \mathrm{H}), 7.39(\mathrm{~s}, 1 \mathrm{H})$, $6.94(\mathrm{~d}, J=7.7 \mathrm{~Hz}, 1 \mathrm{H}), 6.49(\mathrm{~d}, J=4.7 \mathrm{~Hz}, 1 \mathrm{H}), 4.18(\mathrm{~s}, 2 \mathrm{H}), 3.95(\mathrm{~s}, 3 \mathrm{H}), 2.45(\mathrm{~d}, J=6.7 \mathrm{~Hz}$, 2H), 2.42-2.22 (s, 8H), $2.15(\mathrm{~s}, 3 \mathrm{H}), 1.97(\mathrm{~d}, J=5.9 \mathrm{~Hz}, 2 \mathrm{H}) .{ }^{13} \mathrm{C}$ NMR (101 MHz, DMSO) $\delta 169.14$, $160.02,159.18,154.75,152.30,151.97,149.63,148.81,147.69,146.40,142.87,141.78,137.07,129.72(2 \mathrm{C})$, $128.62,124.30,121.35(2 \mathrm{C}), 120.52,116.67,114.50,108.79,108.57,102.14,99.08,66.74,55.80,54.62(2 \mathrm{C})$, 54.27, 52.53(2C), 45.52, 26.02. TOF MS ES+ $(m / z):(\mathrm{M}+\mathrm{H})^{+}$, calcd for $\mathrm{C}_{35} \mathrm{H}_{35} \mathrm{FN}_{6} \mathrm{O}_{5}: 639.2731$, found, 639.2689 .

\subsection{Cytotoxicity Assay In Vitro}

The cytotoxic activities of target compounds (22-53) were evaluated with A549, HepG2, and MCF-7 cell lines by the standard MTT assay in vitro, with compounds c-MET inhibitors Foretinib as positive control. The cancer cell lines were cultured in minimum essential medium (MEM) supplement with $10 \%$ fetal bovine serum (FBS). Approximately $4 \times 103$ cells, suspended in MEM medium, were plated onto each well of a 96-well plate and incubated in $5 \% \mathrm{CO}_{2}$ at $37{ }^{\circ} \mathrm{C}$ for $24 \mathrm{~h}$. The test compounds at indicated final concentrations were added to the culture medium and the cell cultures were continued for $72 \mathrm{~h}$. Fresh MTT was added to each well at a terminal concentration of $5 \mu \mathrm{g} / \mathrm{mL}$ and incubated with cells at $37^{\circ} \mathrm{C}$ for $4 \mathrm{~h}$. The formazan crystals were dissolved in $100 \mu \mathrm{L}$ DMSO each well, and the absorbency at $492 \mathrm{~nm}$ (for absorbance of MTT formazan) and $630 \mathrm{~nm}$ (for the reference wavelength) was measured with the ELISA reader. All of the compounds were tested two times in each of the cell lines. The results expressed as inhibition rates or $\mathrm{IC}_{50}$ (half-maximal inhibitory concentration) were the averages of two determinations and calculated by using the Bacus Laboratories Incorporated Slide Scanner (Bliss) software (the Bacus Laboratories Inc. Slide Scanner (BLISS) system, Lombard, IL, USA). [20].

\subsection{Tyrosine Kinases Assay In Vitro}

The selected compounds (22-53) are tested for their activity against c-Met Tyrosine kinases through the mobility shift assay [8,9]. All kinase assays were performed in 96-well plates in a $50 \mu \mathrm{L}$ reaction volume. The kinase buffer contains $50 \mathrm{mM}$ HEPES, pH 7.5, $10 \mathrm{mM} \mathrm{MgCl}$, 0.0015\% Brij-35 and 2 mM DTT. The stop buffer contains $100 \mathrm{mM} \mathrm{HEPES,} \mathrm{pH} \mathrm{7.5,} \mathrm{0.015 \%} \mathrm{Brij-35,} \mathrm{0.2 \%} \mathrm{Coating}$ Reagent 3 and $50 \mathrm{mM}$ EDTA. Dilute the compounds to $500 \mu \mathrm{M}$ by $100 \% \mathrm{DMSO}$, then transfer $10 \mu \mathrm{L}$ of compound to a new 96-well plate as the intermediate plate, add $90 \mu \mathrm{L}$ kinase buffer to each well. Transfer $5 \mu \mathrm{L}$ of each well of the intermediate plate to 384-well plates. The following amounts of enzyme and substrate were used per well: kinase base buffer, FAM-labeled peptide, ATP and enzyme solution. Wells containing the substrate, enzyme, DMSO without compound were used as DMSO control. Wells containing just the substrate without enzyme were used as low control. Incubate at room temperature for $10 \mathrm{~min}$. Add $10 \mu \mathrm{L}$ peptide solution to each well. Incubate at $28{ }^{\circ} \mathrm{C}$ for specified 
period of time and stop reaction by $25 \mu \mathrm{L}$ stop buffer. At last collect data on Caliper program and convert conversion values to inhibition values. Percent inhibition $=(\max -\operatorname{conversion}) /(\max -\min )$ $\times$ 100. ' $\mathrm{max}^{\prime}$ stands for DMSO control; ' $m i n$ ' stands for low control [20].

\subsection{Observation of Nuclear Morphology}

The cancer cell lines were cultured in minimum essential medium (MEM) supplement with 10\% fetal bovine serum (FBS). Approximately $2 \times 104$ cells, suspended in MEM medium, were plated onto each well of a 24-well plate and incubated in $5 \% \mathrm{CO}_{2}$ at $37{ }^{\circ} \mathrm{C}$ for $24 \mathrm{~h}$. Then the medium was removed, and $1 \mathrm{~mL}$ drug-free medium and $1 \mathrm{~mL}$ medium with the test compound $7 \mathrm{c}$ at indicated final concentrations were added to each well in control group and test group respectively and the cell cultures were continued for $12 \mathrm{~h}$. While the medium was removed, AO-PBS buffer was added to each well at a terminal concentration of $10 \mu \mathrm{g} / \mathrm{mL}$ and stained in dark for $10 \mathrm{~min}$. Each well was washed with PBS buffer three times and observed under a fluorescence microscope.

\subsection{Docking Studies}

For docking purposes, the three-dimensional structure of the c-Met (PDB code: 3LQ8) was obtained from RCSB Protein Data Bank [10]. Hydrogen atoms were added to the structure allowing for appropriate ionization at physiological $\mathrm{pH}$ and the water was removed. The protonated state of several important residues were adjusted by using AutoDock vina v1.02 in favor of forming reasonable hydrogen bond with the ligand. And using AutoDock vina v1.02 to produce nine ligand conformation, the best molecular conformation was used as a ligand that complex bonding affinity score was $-32.83 \mathrm{kcal} / \mathrm{mol}$. Molecular docking of 53 into the 3D c-Met complex structure (PDB code: 3LQ8) was carried out using the Discovery Studio 3.5, as implemented through the graphical user interface LibDock protocol. Using the receptor-ligand interaction part to defined the 3LQ8 as receptor to show the interaction between the 53 and c-Met, then display the residues that contact with 53 and hide others to produce the Figure $4 \mathrm{a}$ after adding the activity pocket. Follow by point the "show 2D diagram" to obtain the Figure 4b. All calculations were performed on Silicon Graphics workstation [20].

\section{Conclusions}

In summary, we designed and synthesized a series of 6,7-disubstituted-4-phenoxyquinoline derivatives bearing pyridazinone skeleton as potential c-Met kinase inhibitors and evaluated for the $\mathrm{IC}_{50}$ values against three cancer cell lines and enzymatic. Eight of them are equal or more active than positive control Foretinib against one or more cell lines and enzymatic. The most promising compound $\mathbf{5 3}$ showed superior activity to Foretinib, which possessed excellent c-Met kinase inhibition on a single digital nanomolar level $\left(\mathrm{IC}_{50}=0.6 \mathrm{nM}\right)$, and cancer cells of $\mathrm{A} 549\left(\mathrm{IC}_{50}=0.003 \mu \mathrm{M}\right)$, HepG2 $\left(\mathrm{IC}_{50}=0.49 \mu \mathrm{M}\right)$ and MCF-7 $\left(\mathrm{IC}_{50}=0.006 \mu \mathrm{M}\right)$. Moreover, SARs and docking studies indicated that 6,7-disubstituted-4-phenoxyquinoline derivatives bearing pyridazinone were favorable to the activity. What's more, the morpholino group was substituted with other water-soluble substituents to favor this activity. In particular, the 4-methylpiperazinyl group was most active. According to the result of AO single staining, it's claimed that the 53 could induce remarkable apoptosis of HepG2 cells. And further study will be carried out to identify the exact action mechanism in near future.

Author Contributions: X.L. and F.T. synthesized all of novel compounds. Z.X., J.H. and J.K. run the bioassay evaluation and statistics analysis. P.Z. started the project and designed the molecules, and W.Z. wrote the paper.

Funding: We gratefully acknowledge the generous support provided by The National Natural Science Funds (No. 21662014), Outstanding Youth Foundation of Jiangxi, Natural Science Foundation of Jiangxi, China (20171BCB23078), Natural Science Foundation of Jiangxi, China (20171ACB21052 \& 20171BAB215073), Science and Technology Project Founded by the Education Department of Jiangxi Province (GJJ160787), Jiangxi Provincial Key Laboratory of Drug Design and Evaluation (20171BCD40015), Innovative Research Team of Jiangxi Science \& Technology Normal University (2017CXTD002), Graduate Students' Science and Technology Innovation Project of Jiangxi Science \& Technology Normal University (YC2017-X21). This research received no external funding. 
Conflicts of Interest: The authors declare no conflict of interest.

\section{References}

1. Gherardi, E.; Stoker, M. Hepatocytes and scatter factor. Nature 1990, 346, 228. [CrossRef] [PubMed]

2. Trovato, M.; Grosso, M.; Vitarelli, E.; Benvenga, S.; Trimarchi, F.; Barresi, G. Immunoexpression of the hepatocyte growth factor (HGF), HGF-receptor (c-met) and STAT3 on placental tissues from malformed fetuses. Histol. Histopathol. 2002, 17, 691-698. [PubMed]

3. Gherardi, E.; Birchmeier, W.; Birchmeier, C.; Vande Woude, G. Targeting MET in cancer: Rationale and progress. Nat. Rev. Cancer 2012, 12, 89-103. [CrossRef] [PubMed]

4. Ugolini, A.; Kenigsberg, M.; Rak, A.; Vallée, F.; Houtmann, J.; Lowinski, M.; Capdevila, C.; Khider, J.; Albert, E.; Martinet, N.; et al. Discovery, pharmacokinetic and pharmacological properties of the potent and selective MET kinase inhibitor, 1-\{6-[6-(4-Fluoro-phenyl)-[1,2,4]triazolo[4,3-b]pyridazin-3ylsulfanyl]-benzothiazol-2-yl-3-(2-morpholin-4-yl-ethyl)-urea (SAR125844). J. Med. Chem. 2016, 59, 7066-7074. [CrossRef] [PubMed]

5. Jeffers, M.; Rong, S.; Anver, M.; Vande Woude, G.F. Autocrine hepatocyte growth factor/scatter factor-Met signaling induces transformation and the invasive/metastastic phenotype in C127 cells. Oncogene 1996, 13, 853-856. [PubMed]

6. Takayama, H.; LaRochelle, W.J.; Sharp, R.; Otsuka, T.; Kriebel, P.; Anver, M.; Aaronson, S.A.; Merlino, G. Diverse tumorigenesis associated with aberrant development in mice overexpressing hepatocyte growth factor/scatter factor. Proc. Natl. Acad. Sci. USA 1997, 94, 701-706. [CrossRef] [PubMed]

7. Otsuka, T.; Takayama, H.; Sharp, R.; Celli, G.; LaRochelle, W.J.; Bottaro, D.P.; Ellmore, N.; Vieira, W.; Owens, J.W.; Anver, M.; et al. c-Met autocrine activation induces development of malignant melanoma and acquisition of the metastatic phenotype. Cancer Res. 1998, 58, 5157. [PubMed]

8. Aguirre Ghiso, J.A.; Alonso, D.F.; Farías, E.F.; Gomez, D.E.; de Kier Joffè, E.B. Deregulation of the signaling pathways controlling urokinase production. Its relationship with the invasive phenotype. FEBS J. 1999, 263, 295-304. [CrossRef]

9. Parr, C.; Watkins, G.; Mansel, R.E.; Jiang, W.G. The hepatocyte growth factor regulatory factors in human breast cancer. Clin. Cancer Res. 2004, 10 Pt 1, 202-211. [CrossRef] [PubMed]

10. Straussman, R.; Morikawa, T.; Shee, K.; Barzily-Rokni, M.; Qian, Z.R.; Du, J.; Davis, A.; Mongare, M.M.; Gould, J.; Frederick, D.T.; et al. Tumour micro-environment elicits innate resistance to RAF inhibitors through HGF secretion. Nature 2012, 487, 500-504. [CrossRef] [PubMed]

11. Bean, J.; Brennan, C.; Shih, J.Y.; Riely, G.; Viale, A.; Wang, L.; Chitale, D.; Motoi, N.; Szoke, J.; Broderick, S.; et al. MET amplification occurs with or without T790M mutations in EGFR mutant lung tumors with acquired resistance to gefitinib or erlotinib. Proc. Natl. Acad. Sci. USA 2007, 104, 20932-20937. [CrossRef] [PubMed]

12. You, W.K.; Sennino, B.; Williamson, C.W.; Falcón, B.; Hashizume, H.; Yao, L.C.; Aftab, D.T.; McDonald, D.M. VEGF and c-Met blockade amplify angiogenesis inhibition in pancreatic islet cancer. Cancer Res. 2011, 71, 4758-4768. [CrossRef] [PubMed]

13. Moffat, D.F.C.; Batchellor, M.J.; Brookings, D.C.; David, P.D. 4-Aminosulfonylquinazolines and-Quinolines as Tyrosine Kinase Inhibitors. WO Patent 2002/087587 A2, 7 November 2002.

14. Fujita, H.; Miyadera, K.; Kato, M.; Fujioka, Y.; Ochiiwa, H.; Huang, J.; Ito, K.; Aoyagi, Y.; Takenaka, T.; Suzuki, T; et al. The novel VEGF receptor/MET-targeted kinase inhibitor TAS-115 has marked in vivo antitumor properties and a favorable tolerability profile. Mol. Cancer Ther. 2013, 12, 2685-2696. [CrossRef] [PubMed]

15. Takigawa, N. MET-inhibitors meet MET mutations in lung cancer. Transl. Cancer Res. 2016, 5, S1248-S1254. [CrossRef]

16. Yan, S.B.; Peek, V.L.; Ajamie, R.; Buchanan, S.G.; Graff, J.R.; Heidler, S.A.; Hui, Y.H.; Huss, K.L.; Konicek, B.W.; Manro, J.R.; et al. LY2801653 is an orally bioavailable multi-kinase inhibitor with potent activity against MET, MST1R, and other oncoproteins, and displays anti-tumor activities in mouse xenograft models. Investig. New Drugs 2013, 31, 833-844. [CrossRef] [PubMed] 
17. Werner, T.; Heist, R.; Carvajal, R.; Adkins, D.; Alva, A.; Goel, S.; Hong, D.; Bazhenova, L.; Saleh, M.; Siegel, R.; et al. P2. 06-001 A Study of MGCD516, a Receptor Tyrosine Kinase (RTK) Inhibitor, in Molecularly Selected Patients with NSCLC or Other Advanced Solid Tumors: Topic: Phase I Trials. J. Thorac. Oncol. 2017, 12, S1068-S1069. [CrossRef]

18. Smith, B.D.; Kaufman, M.D.; Leary, C.B.; Turner, B.A.; Wise, S.C.; Ahn, Y.M.; Booth, R.J.; Caldwell, T.M.; Ensinger, C.L.; Hood, M.M.; et al. Altiratinib Inhibits Tumor Growth, Invasion, Angiogenesis, and Microenvironment-Mediated Drug Resistance via Balanced Inhibition of MET, TIE2, and VEGFR2. Mol. Cancer Ther. 2015, 14, 2023-2034. [CrossRef] [PubMed]

19. Li, S.; Zhao, Y.; Wang, K.; Gao, Y.; Han, J.; Cui, B.; Gong, P. Discovery of novel 4-(2-fluorophenoxy)quinoline derivatives bearing 4-oxo-1,4-dihydrocinnoline-3-carboxamide moiety as c-Met kinase inhibitors. Bioorg. Med. Chem. 2013, 21, 2843-2855. [CrossRef] [PubMed]

20. Tang, Q.; Wang, L.; Duan, Y.; Wang, W.; Huang, S.; Zhi, J.; Zhu, W.; Wang, P.; Luo, R.; Zheng, P. Discovery of novel 7-azaindole derivatives bearing dihydropyridazine moiety as c-Met kinase inhibitors. Eur. J. Med. Chem. 2017, 133, 97-106. [CrossRef] [PubMed]

21. Kataoka, Y.; Mukohara, T.; Tomioka, H.; Funakoshi, Y.; Kiyota, N.; Fujiwara, Y.; Yashiro, M.; Hirakawa, K.; Hirai, M.; Minami, H. Foretinib (GSK1363089), a multi-kinase inhibitor of MET and VEGFRs, inhibits growth of gastric cancer cell lines by blocking inter-receptor tyrosine kinase networks. Investig. New Drugs 2012, 30, 1352-1360. [CrossRef] [PubMed]

22. Li, X.; Zhao, T.; Chen, Q.; Li, P.; Wang, K.; Zhong, M.; Wei, J.; Wu, D.; Weief, B.; Zhu, H. Flexible all solid-state supercapacitors based on chemical vapor deposition derived graphene fibers. Phys. Chem. Chem. Phys. 2013, 15, 17752-17757. [CrossRef] [PubMed]

Sample Availability: Samples of the compounds $\mathbf{2 2 - 5 3}$ are available from the authors.

(C) 2018 by the authors. Licensee MDPI, Basel, Switzerland. This article is an open access article distributed under the terms and conditions of the Creative Commons Attribution (CC BY) license (http:// creativecommons.org/licenses/by/4.0/). 